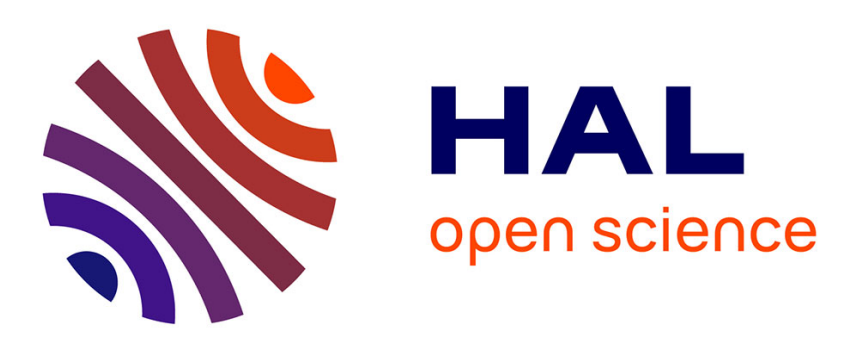

\title{
Investigation of localized necking in substrate-supported metal layers: comparison of bifurcation and imperfection analyses
}

Mohamed Ben Bettaieb, Farid Abed-Meraim

\section{- To cite this version:}

Mohamed Ben Bettaieb, Farid Abed-Meraim. Investigation of localized necking in substrate-supported metal layers: comparison of bifurcation and imperfection analyses. International Journal of Plasticity, 2015, 65, pp.168-190. 10.1016/j.ijplas.2014.09.003 . hal-01201806

\section{HAL Id: hal-01201806 \\ https://hal.science/hal-01201806}

Submitted on 18 Sep 2015

HAL is a multi-disciplinary open access archive for the deposit and dissemination of scientific research documents, whether they are published or not. The documents may come from teaching and research institutions in France or abroad, or from public or private research centers.
L'archive ouverte pluridisciplinaire HAL, est destinée au dépôt et à la diffusion de documents scientifiques de niveau recherche, publiés ou non, émanant des établissements d'enseignement et de recherche français ou étrangers, des laboratoires publics ou privés. 


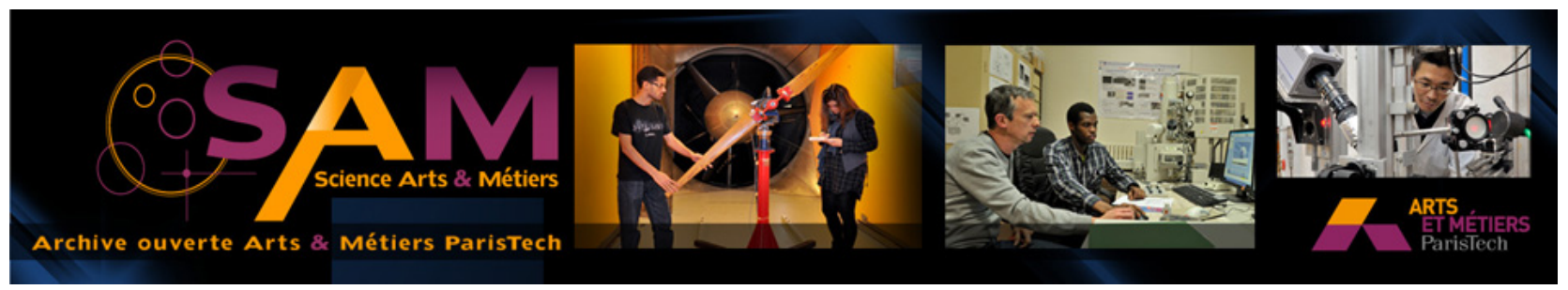

\section{Science Arts \& Métiers (SAM)}

is an open access repository that collects the work of Arts et Métiers ParisTech researchers and makes it freely available over the web where possible.

This is an author-deposited version published in: http://sam.ensam.eu

Handle ID: .http://hdl.handle.net/10985/10047

\section{To cite this version :}

Mohamed BEN BETTAIEB, Farid ABED-MERAIM - Investigation of localized necking in substratesupported metal layers: comparison of bifurcation and imperfection analyses - International Journal of Plasticity - Vol. 65, p.168-190 - 2015 


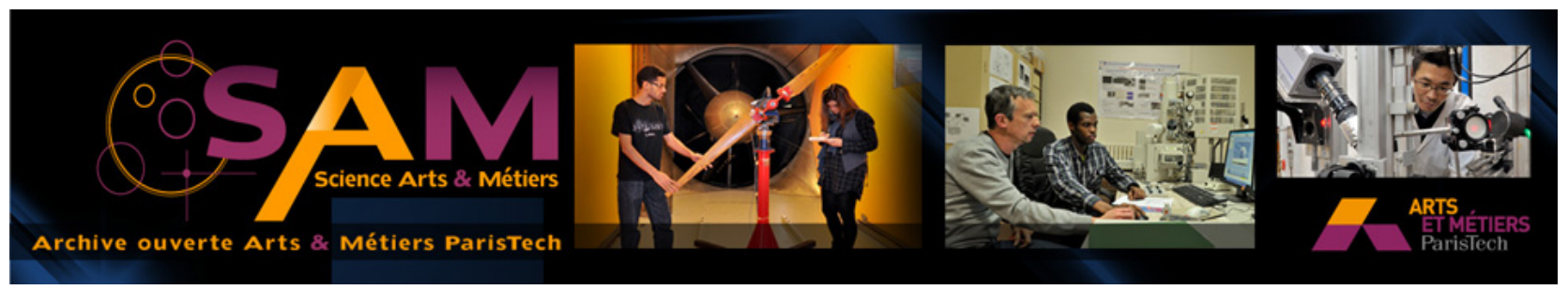

Science Arts \& Métiers (SAM)

is an open access repository that collects the work of Arts et Métiers ParisTech researchers and makes it freely available over the web where possible.

This is an author-deposited version published in: http://sam.ensam.eu Handle ID: .http://hdl.handle.net/null

\section{To cite this version :}

Mohamed BEN BETTAIEB, Farid ABED-MERAIM - Investigation of localized necking in substratesupported metal layers: comparison of bifurcation and imperfection analyses - International Journal of Plasticity - Vol. 65, p.168-190 - 2014 


\title{
Investigation of localized necking in substrate-supported metal layers: comparison of bifurcation and imperfection analyses
}

\author{
Mohamed Ben Bettaieb*, Farid Abed-Meraim \\ LEM3, UMR CNRS 7239 - Arts et Métiers ParisTech, 4 rue Augustin Fresnel, 57078 Metz Cedex 3, France \\ DAMAS, Laboratory of Excellence on Design of Alloy Metals for low-mAss Structures, Université de Lorraine, \\ France
}

\begin{abstract}
Localized necking is often considered as precursor to failure in metal components. In modern technologies, functional components (e.g., in flexible electronic devices) may be affected by this necking phenomenon, and to avoid the occurrence of strain localization, elastomer substrates are bonded to the metal layers. This paper proposes an investigation of the development of localized necking in both freestanding metal layers and elastomer/metal bilayers. Finite strain versions of both rigid-plastic flow theory and deformation theory of plasticity are employed to model the mechanical response of the metal layer. For the elastomer, a neo-Hookean constitutive law is considered. Localized necking is predicted using both bifurcation (whenever possible) and Marciniak-Kuczynski analyses. A variety of numerical results are presented, which pertain to the prediction of localized necking in freestanding metal layers and metal/substrate bilayers. The effects of the constitutive framework and the presence of an elastomer substrate on strain localization predictions have been specifically highlighted. It is demonstrated that the addition of an elastomer layer can retard significantly the occurrence of localized necking. It is also demonstrated that the results of the Marciniak-Kuczynski analysis tend towards the bifurcation predictions in the limit of a vanishing size for the geometric imperfection.
\end{abstract}

Keywords: substrate-supported metals, localized necking, deformation theory, flow theory, neoHookean model, bifurcation and imperfection analyses 


\section{Introduction}

Nowadays, necking limit prediction of metal sheets or thin plates represents an ambitious challenge both for the sheet metal forming industry and for the design of functional components in electronic devices. In the well-known experiment of a tensile test on a metal specimen, the deformation develops mainly through three successive stages: (i) a homogeneous deformation; (ii) a progressively concentrating strain under a constant or smoothly decreasing load (diffuse necking), and (iii) an abrupt strain localization (localized necking) under a rapid load decrease. The onset of localized necking represents the ultimate deformation that a stretched metal sheet can undergo, since this phenomenon is often precursor to material failure. Probably, the most common representation of this limit is through the concept of forming limit diagram (FLD). Note that this concept was initially introduced in the beginning of the sixties by Keeler and Backofen (1963), in the range of positive minor principal strains (i.e. $\varepsilon_{2}>0$ ), and Goodwin (1968) (extending Keeler's work to negative minor principal strains, i.e. $\left.\varepsilon_{2}<0\right)$. In the literature, a large amount of studies have been devoted to the experimental and numerical determination of FLDs for sheet metals with different material properties (Smith and Lee, 1998; Narayanasamy and Sathiya Narayanan, 2005; Strano and Colosimo, 2006; Khan and Baig, 2011; Zhang and Wang, 2012; Li et al., 2013). In the vast majority of these studies, attention was restricted to freestanding metal sheets. However, the need for increasing the ductility of metal components has induced an impetus to develop alternative and more complex materials such as substrate-metal bilayers. Indeed, supporting a metal layer by an elastomer substrate has proven to significantly improve its ductility (Chiu et al., 1994; Hommel and Kraft, 2001; Alaca et al., 2002) and to enhance its energy absorption (Xue and Hutchinson, 2007, 2008). In an industrial context, substratesupported metal layers are being used in a variety of flexible electronic devices such as conductors and interconnects (Lacour et al., 2005; Cotton et al., 2009; Cordill et al., 2010; Graudejus et al., 2012). Despite the increasing industrial interest in elastomer/metal bilayers, there is still a need for further studies for an in-depth understanding of the corresponding strain localization conditions. In this paper, an attempt is made to investigate the impact of an elastomer layer, bonded to a metal sheet or plate, on the shape and location of the associated FLD in the in-plane principal strain space. Note that the concept of FLD and associated terminology, more commonly adopted in the literature for a freestanding metal layer, is extended here to a metal/substrate bilayer. In the related literature (Xue and Hutchinson, 2007; Jia and Li, 2013), a similar terminology, namely "necking limit diagram", is preferred instead.

Due to the complexity of the experimental determination of the FLD and its relatively high cost, a number of theoretical and/or numerical models have been set up. These alternative approaches require the use of a criterion, to predict the occurrence of strain localization, along with a constitutive law to describe the evolution of the mechanical state of the studied material. The onset of plastic flow localization may occur as a bifurcation from a homogeneous deformation state or it may be triggered by some assumed initial imperfection. Accordingly, two main classes of strain localization criteria, which will also be used in this paper, can be found in the literature: 
- Imperfection approach:

This approach postulates the existence of an initial imperfection in the form of a narrow band across the studied metal layer. This imperfection may be assumed as local variations in thickness (geometric imperfection) or in plastic properties (material imperfection), which will affect the plastic flow and therefore influence the strain localization occurrence. This approach was initially introduced by Marciniak and Kuczynski (1967), which will be referred to hereafter as the $\mathrm{M}-\mathrm{K}$ analysis. In this pioneering work, the authors introduced an initial geometric imperfection in the sheet plane in the form of a groove or band. During in-plane biaxial stretching, the plastic deformation concentrates in the band more than in the rest of the sheet, leading thus to localized thinning in the band. In its initial version, the $\mathrm{M}-\mathrm{K}$ analysis was restricted to a groove perpendicular to the major strain direction, thus limiting the prediction of FLD to the right-hand side of the FLD $\left(\varepsilon_{2}>0\right)$. To overcome this limitation, Hutchinson and Neale (1978b) proposed an extension of this approach. This extension covers the full range of the FLD by considering all possible initial orientations of the groove and selecting the lowest value of the major strain at the onset of localized necking as the limit strain. The accuracy in the prediction of the FLD has increased over the years by improvement of the constitutive modeling (see, e.g., Barlat, 1989; Eyckens et al., 2009). In spite of the over-sensitivity of its predictions to the initial imperfection value (see, e.g., Baudelet, 1984), the M-K analysis has attracted a great deal of attention, due to its pragmatic character.

- Bifurcation analysis:

In many ductile materials, zones of localized deformation are commonly observed prior to failure, which are considered as a result of instability in the constitutive description of homogeneous deformation. These localization bands induce a macroscopic discontinuity in the velocity gradient of the deforming material and often signal the inception of failure. In addition to its sound mathematical background, the bifurcation theory does not require any fitting parameter, such as the initial imperfection needed in the $\mathrm{M}-\mathrm{K}$ analysis. The bifurcation analysis was initially proposed by Hill (1952) in the case of flow theory of plasticity (rigid-plastic material) within the framework of generalized plane stress. His theory predicts that localized necking occurs along a line of zero extension and is therefore restricted to negative minor strain values (i.e., the left-hand side of the FLD). For elasto-plastic material models with smooth yield surface and associative plasticity, it has been shown (see, e.g., Rice, 1976) that the bifurcation approach does not predict localized necking at a realistic stress level under positive in-plane biaxial stretching (i.e., in the right-hand side of the FLD). In order to overcome this limitation, the introduction of some destabilizing effects is required to promote material instability. To this end, a number of authors suggested that the subsequent yield surfaces of the material would develop a vertex-like structure during continued deformation. The development of such a destabilizing vertex may be due to the application of the deformation theory of plasticity (Stören and Rice, 1975; Hutchinson and Neale, 1978b, 1981), or to the use of the Schmid law within the framework of crystal plasticity (Franz et al., 2009, 2013). Material instability may also be due to 
some softening behavior introduced in the constitutive modeling through coupling with damage (see, e.g., Rudnicki and Rice, 1975; Saje et al., 1982, for pressure-sensitive void containing materials, or Haddag et al., 2009, within the framework of continuum damage mechanics).

The main objective of this paper is to extend the earlier contributions of Hutchinson and Neale (1978b), Xue and Hutchinson (2007) and Jia and Li (2013). In the first of this series of works, Hutchinson and Neale (1978b) extensively studied the necking limit of freestanding metal sheets (or layers) using the bifurcation and the imperfection approaches. For each of these two localization approaches, a rigid-plastic finite strain version of the $J_{2}$ flow theory (designated shortly in what follows as "flow theory") and of the $J_{2}$ deformation theory of plasticity (called shortly hereafter "deformation theory") were used to model the mechanical behavior of the metal sheet. Our current study extends Hutchinson and Neale's (1978b) work to the case of substrate-metal bilayers. The constitutive models of the metal layer are taken the same as those used in Hutchinson and Neale (1978b). However, the behavior of the substrate is modeled by a neo-Hookean constitutive law. In Xue and Hutchinson (2007), the necking limit of elastomer-supported metal layers was investigated using only the bifurcation theory, with the metal layer modeled only by the deformation theory of plasticity. The predictions of the latter study were confined to the range of positive minor strain. Jia and $\mathrm{Li}$ (2013) extended the analysis of Xue and Hutchinson (2007) to the range of negative minor strain. In our current contribution, we enlarge the above two earlier works by adding another localization criterion (the imperfection approach) and another constitutive framework for the metal layer (the flow theory). The developed approaches and associated numerical tools can be used in a wide range of applications. They can be applied to evaluate the immunity of very thin bilayers (for example in micro-electronic applications) as well as thicker bilayers (used for example in mechanical components). The main result of our paper is that the addition of an elastomer layer can retard significantly the occurrence of localized necking. It is also demonstrated that the results of the Marciniak-Kuczynski analysis tend towards the bifurcation predictions in the limit of a vanishing size for the geometric imperfection, both for an all-metal single layer and for a metal/substrate bilayer.

The remainder of the paper is organized as follows:

- Section 2 outlines the constitutive equations used to model the metal and elastomer layers.

- Section 3 details the localization criteria employed to predict localized necking in the bilayer.

- Section 4 deals with the numerical and algorithmic developments used to couple the constitutive equations with the localization criteria.

- The various numerical predictions are presented in Section 5. These results are discussed in details and compared with some reference results taken from the literature.

\section{Notations, conventions and abbreviations}

The derivations presented in this paper are carried out using classic conventions. Note that the assorted notations can be combined, while additional notations will be clarified as needed following related equations. 


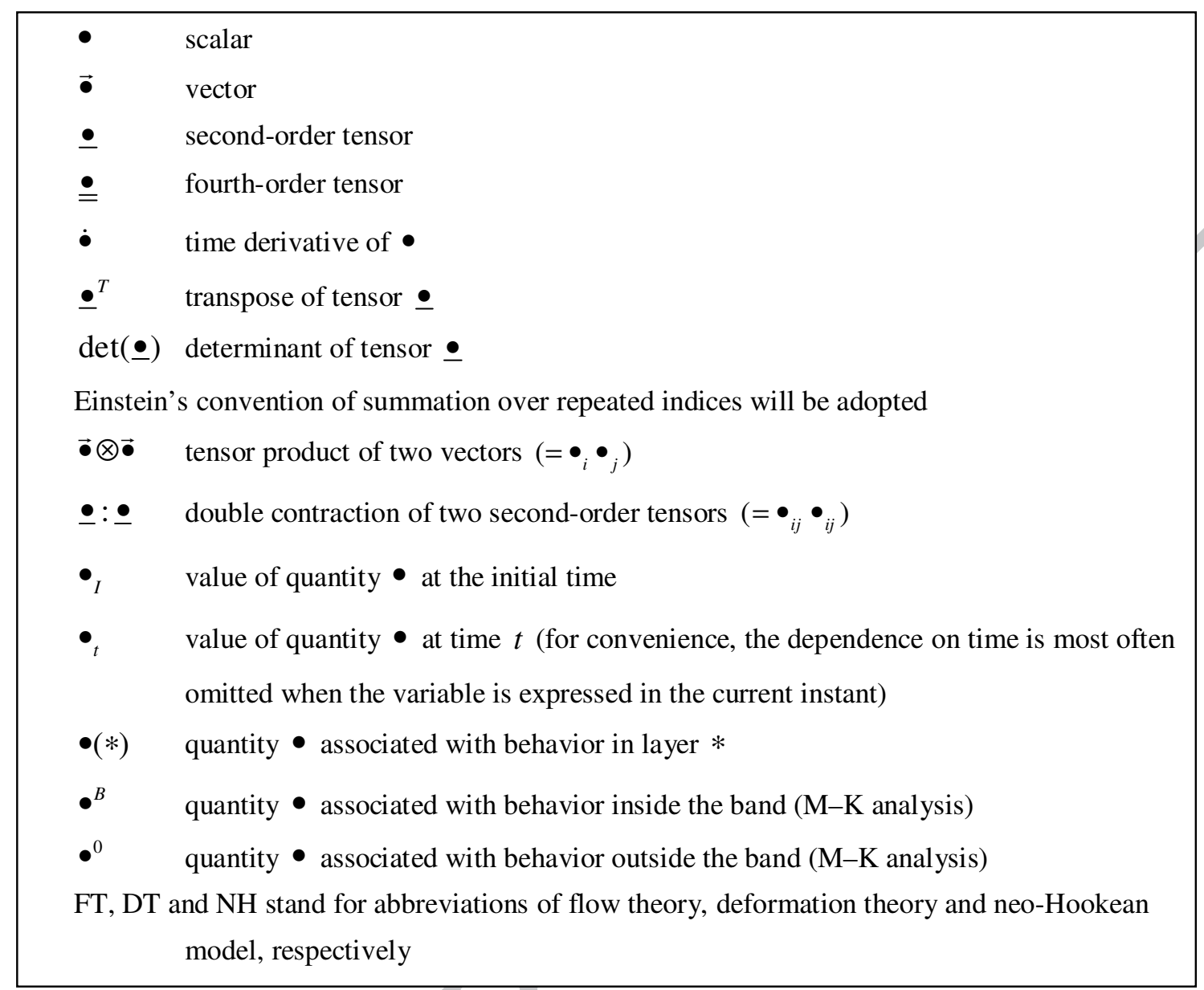

\section{Constitutive equations}

Consider a quasi-static deformation of a thin metal/substrate bilayer under in-plane biaxial stretching. We define an orthogonal Cartesian coordinate system $\left(x_{1}, x_{2}, x_{3}\right)$, which is fixed to the bilayer (Fig. 1), with axes $\left(x_{1}, x_{2}\right)$ lying in the bilayer plane, and axis $x_{3}$ normal to this plane.

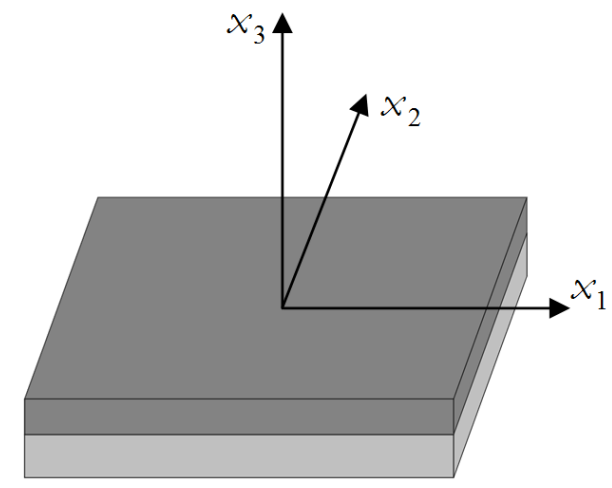

Fig. 1. Geometry of the bilayer and Cartesian coordinates. 
The following choices and assumptions are considered in the modeling of the bilayer behavior:

- Two different constitutive models are employed in this study to describe the mechanical behavior of the metal layer: rigid-plastic flow theory and deformation theory of plasticity.

- Strain localization occurs at relatively large strains so that the elasticity of the metal layer can be neglected (i.e., the mechanical behavior is assumed to be rigid-plastic).

- The mechanical response of the elastomer substrate is defined by a neo-Hookean behavior model.

- The metal and the elastomer layers are assumed to be isotropic, incompressible and strain-rate independent.

- The materials composing the bilayer remain bonded and are such that material damage does not occur prior to necking.

- The constitutive framework adopted here is of phenomenological nature and thus unaffected by microstructural size effects.

Firstly, the constitutive equations are established in the general three-dimensional case. Secondly, the incompressibility condition and plane-stress assumption are employed to adapt the constitutive equations to a two-dimensional formulation (i.e., in the plane of the bilayer). For convenience, a ratetype formulation is used to express the constitutive equations of the flow theory (with the rate of Eulerian strain measure). However, a "total" formulation is used in the development of the constitutive equations of both the deformation theory and the neo-Hookean model (with Lagrangian strain measures).

\subsection{Flow theory of plasticity}

Associative plasticity is assumed and, accordingly, the Eulerian strain rate tensor $\dot{\varepsilon}$ (the symmetric part of the velocity gradient $\underline{G}$ ) is given by the normality flow rule:

$$
\dot{\varepsilon}=\dot{\lambda} \frac{\partial \sigma_{e q}}{\partial \underline{\sigma}}
$$

Here, $\underline{\sigma}$ is the Cauchy stress tensor, $\sigma_{e q}$ is the equivalent stress (in the sense of von Mises), equal to $\sqrt{(3 / 2) \underline{S}: \underline{S}}$ ( $\underline{S}$ being the deviatoric part of $\underline{\sigma}$ ), and $\dot{\lambda}$ is the plastic multiplier (equal to the equivalent strain rate $\dot{\varepsilon}_{e q}$ for associative rigid-plasticity).

The rigid-plastic constitutive law can then be derived from Eq. (1) as:

$$
\underline{\dot{\varepsilon}}=\frac{3}{2} \frac{\dot{\varepsilon}_{e q}}{\sigma_{e q}} \underline{S} \Leftrightarrow \underline{S}=\frac{2}{3} \frac{\sigma_{e q}}{\dot{\varepsilon}_{e q}} \underline{\dot{\varepsilon}}
$$




\subsection{Deformation theory of plasticity}

\subsubsection{Some preliminary concepts}

The deformation gradient $\underline{F}$ is polarly decomposed into the left Cauchy-Green stretch tensor $\underline{V}$ and the proper orthogonal rotation tensor $\underline{R}$ :

$$
\underline{F}=\underline{V} \underline{R} \Rightarrow \underline{F}^{T}=\underline{V}^{2}
$$

$\underline{V}$ can itself be decomposed in the following form:

$$
\underline{V}=\underline{M} \underline{\lambda} \underline{M}^{T}
$$

where $\underline{\lambda}$ is a diagonal tensor whose diagonal terms $\left(\lambda_{1}, \lambda_{2}, \lambda_{3}\right)$ define the principal stretches (the principal values of tensor $\underline{V}$ ), while $\underline{M}$ is an orthogonal tensor defining the orientation of the principal strain directions relative to the Cartesian coordinate system $\left(x_{1}, x_{2}, x_{3}\right)$.

\subsubsection{Formulation of the deformation theory in the frame of principal strain directions}

The deformation theory was initially developed in Hill (1950) for isotropic materials under small strain assumptions. It was then extended to the case of finite strains in Hutchinson and Neale (1978b, 1981). In these different works, the constitutive equations are expressed in the frame of the principal strain directions, which coincides with the frame of principal stresses (as a consequence of isotropy). In this frame, the deformation gradient $\underline{F}$ is reduced to the tensor $\underline{\lambda}$. Accordingly, the most appropriate strain measure appears to be the logarithmic strain $\varepsilon_{i}$ defined by the following relation:

$$
\varepsilon_{i}=\ln \lambda_{i} \quad ; \quad i=1,2,3
$$

The basic equations of the finite strain deformation theory provide relations between the logarithmic strains $\varepsilon_{i}$ and the principal deviatoric stresses $S_{i}$ as follows:

$$
S_{i}=\frac{2}{3} \frac{\sigma_{e q}}{\varepsilon_{e q}} \varepsilon_{i} \quad ; \quad i=1,2,3
$$

where $\sigma_{e q}=\sqrt{3 S_{i} S_{i} / 2}$ and $\varepsilon_{e q}=\sqrt{2 \varepsilon_{i} \varepsilon_{i} / 3}$ are, respectively, the equivalent stress and the equivalent strain.

The deformation theory provides the same stress-strain response as the flow theory for a proportional and monotonic loading history. In this case, Eqs. (2) and (6) become equivalent. In situations involving a proportional or nearly proportional monotonic loading history, deformation theory may be considered as an acceptable plasticity theory. In other loading conditions (strongly non-proportional loading, unloading), several objections were raised concerning the use of the deformation theory. In contrast, the applicability of flow theory does not require such restrictions.

As elasticity in neglected, $\sigma_{e q}$ (resp. $\varepsilon_{e q}$ ) is always equal to the effective stress $\sigma_{e}$ (resp. effective strain $\varepsilon_{e}$ ). Therefore, Eq. (6) is equivalent to:

$$
S_{i}=\frac{2}{3} \frac{\sigma_{e}}{\varepsilon_{e}} \varepsilon_{i}=\frac{2}{3} E_{S} \varepsilon_{i} \quad ; \quad i=1,2,3
$$


where $E_{S}$ is the secant modulus.

\subsubsection{Extension of the deformation theory to general Cartesian coordinates}

Expression (7) is sufficient to carry out the bifurcation analysis, especially when the principal strain directions coincide with the Cartesian coordinate axes $\left(x_{1}, x_{2}, x_{3}\right)$. However when the two frames do not coincide, Eq. (7) must be generalized. To this end, let us introduce the scalar strain energy function:

$$
W=\int \sigma_{e} d \varepsilon_{e}=\int \sqrt{(3 / 2) \underline{S}: \underline{S}} d \varepsilon_{e}
$$

which is equivalent (after straightforward calculation) to:

$$
W=\frac{1}{3} \int E_{S}(\Pi) d \Pi
$$

where the scalar $\Pi$ is defined as a function of $\ln \underline{V}$ (the natural logarithmic of tensor $\underline{V}$ ):

$$
\Pi=\ln (\underline{V}): \ln (\underline{V})=\ln ^{2}\left(\lambda_{1}\right)+\ln ^{2}\left(\lambda_{2}\right)+\ln ^{2}\left(\lambda_{3}\right)=(3 / 2) \varepsilon_{e}^{2}
$$

and $E_{S}(\Pi)$ is defined by:

$$
E_{S}(\Pi)=\frac{3}{2} \sqrt{\frac{S}{\Pi}: \frac{S}{\Pi}}
$$

From Eq. (11), we obtain:

$$
\underline{S}=\frac{2}{3} E_{S} \ln \underline{V}
$$

Eq. (12) is regarded as a tensorial generalization of constitutive equation (7), the former being applicable in any arbitrary coordinate system. It is considered to be the hyperelastic version of the deformation theory. However, a hypoelastic (path dependent) version of the deformation theory was developed by Stören and Rice (1975). In this hypoelastic version, the constitutive equation, which is the counterpart of Eq. (12), is given by:

$$
\underline{S}=\frac{2}{3} E_{S} \underline{\varepsilon} \quad ; \quad \underline{\varepsilon}=\int \underline{\dot{\varepsilon}} d t
$$

When the principal axes of strain do not rotate with the material, both versions coincide.

\subsection{Neo-Hookean model}

The neo-Hookean model adopted in this paper to describe the mechanical behavior of the elastomer substrate is defined by the following constitutive equation (Hunter, 1979; Rajagopal, 1998):

$$
\underline{S}=\mu \underline{V}^{2}
$$

where $\mu$ is the shear modulus.

This constitutive law is selected among other models (Baghani et al., 2012; Bouvard et al., 2013; Uchida and Tada, 2013; Ayoub et al., 2014), because it furnishes a valid description for the finite 
elastic behavior of many real rubber-like materials, provided that the deformations are not too extreme.

\subsection{Plane-stress formulation}

The constitutive equations (2), (12) and (14), previously derived in terms of the deviatoric stress tensor $\underline{S}$, can be rewritten in terms of the Cauchy stress tensor $\underline{\sigma}$ using the relation:

$$
\underline{\sigma}=\underline{S}+p \underline{I d}
$$

where $\underline{I d}$ is the second-order identity tensor and $p$ is the hydrostatic pressure.

Consistent with several literature works and because the bilayer is assumed to be thin, the assumption of generalized plane stress will be adopted in both the bifurcation and the $\mathrm{M}-\mathrm{K}$ analyses. Hutchinson et al. (1978a) have proven the validity of such an assumption in the case of thin media. Under this condition, the stress components normal to the bilayer $\sigma_{i 3} ; i=1,2,3$ are always zero. Thus, the hydrostatic pressure $p$ can be eliminated from Eq. (15) by means of relation $\sigma_{33}=0$, which gives after straightforward calculation: $p=S_{11}+S_{22}$.

The constitutive equations associated with the flow theory, the deformation theory, and the neoHookean model can be rewritten as:

$$
\underline{\sigma}=\underline{S}+\left(S_{11}+S_{22}\right) \underline{I d}
$$

where the expression of the deviatoric stress tensor $\underline{S}$ has to be substituted, respectively, by:

$$
\underline{S}=\frac{2}{3} \frac{\sigma_{e q}}{\dot{\varepsilon}_{e q}} \dot{\dot{\varepsilon}} \quad ; \quad \underline{S}=\frac{2}{3} E_{S} \ln \underline{V} \quad ; \quad \underline{S}=\mu \underline{V}^{2}
$$

Equation (16) in addition to Eqs. (17) summarize the constitutive equations for the different material models (i.e., flow theory, deformation theory, and neo-Hookean model) in the case of plane-stress conditions. These equations will be coupled with the localization criteria in order to predict the FLDs of substrate-metal bilayers.

\section{Localized necking criteria}

\subsection{Bifurcation theory}

In order to establish the governing equations for the Rice bifurcation criterion, it is first necessary to derive the instantaneous modulus $\underline{L}$ for the different behavior models considered. This modulus relates the Jaumann co-rotational rate of the Cauchy stress tensor $\underline{\sigma}^{J}$ to the strain rate tensor $\underline{\dot{\varepsilon}}$ (symmetric part of the velocity gradient $\underline{G}$ ):

$$
\underline{\sigma}^{J}=\underline{\underline{L}}: \underline{\dot{\varepsilon}}
$$

In the bifurcation analysis, we assume without loss of generality that the direction of the principal stress $\sigma_{11}\left(\right.$ resp. $\left.\sigma_{22}\right)$ is coaxial with the reference axis $x_{1}$ (resp. $x_{2}$ ) of the fixed Cartesian frame. In 
this case, the state of uniform stress considered prior to bifurcation is such that the only non-vanishing stress components are $\sigma_{11}$ and $\sigma_{22}$.

Taking into account the plane-stress condition and the coaxiality of the Cartesian base vectors $\left(x_{1}, x_{2}\right)$ with the principal stress axes $\left(\sigma_{11}, \sigma_{22}\right)$, Eq. (18) can be reduced to:

$$
\left\{\begin{array}{l}
\sigma_{11}^{J}=L_{11} \dot{\varepsilon}_{11}+L_{12} \dot{\varepsilon}_{22} \\
\sigma_{22}^{J}=L_{12} \dot{\varepsilon}_{11}+L_{22} \dot{\varepsilon}_{22} \\
\sigma_{12}^{J}=2 L_{S} \dot{\varepsilon}_{12}
\end{array}\right.
$$

The aim of the following subsections is to explicitly derive the expressions of components $L_{11}, L_{22}, L_{12}$ and $L_{S}$ of the instantaneous modulus $\underline{\underline{L}}$ corresponding to the different constitutive models considered.

\subsubsection{Instantaneous modulus for the flow theory}

Hutchinson and Neale (1978b) provided the expression of the instantaneous modulus in the case of incompressible and isotropic elasto-plastic flow theory:

$$
\left\{\begin{array}{l}
L_{11}=\frac{4}{3} E-\left(E-E_{T}\right)\left(\frac{\sigma_{11}}{\sigma_{e}}\right)^{2} ; L_{22}=\frac{4}{3} E-\left(E-E_{T}\right)\left(\frac{\sigma_{22}}{\sigma_{e}}\right)^{2} \\
L_{12}=\frac{2}{3} E-\left(E-E_{T}\right)\left(\frac{\sigma_{11} \sigma_{22}}{\sigma_{e}^{2}}\right) ; L_{S}=\frac{E}{3}
\end{array}\right.
$$

where $E$ and $E_{T}$ are respectively the Young modulus and the scalar tangent modulus equal to $d \sigma_{e} / d \varepsilon_{e}$.

The rigid-plastic behavior can be considered as a limiting case of the elasto-plastic behavior, when $E \rightarrow+\infty$.

\subsubsection{Instantaneous modulus for the deformation theory}

The instantaneous modulus used for the deformation theory was derived by Hutchinson and Neale (1978b, 1981). This derivation is based on the "principal axes technique" introduced by Hill (1970):

$$
\left\{\begin{array}{l}
L_{11}=\frac{4}{3} E_{S}-\left(E_{S}-E_{T}\right)\left(\frac{\sigma_{11}}{\sigma_{e}}\right)^{2} \quad ; \quad L_{22}=\frac{4}{3} E_{S}-\left(E_{S}-E_{T}\right)\left(\frac{\sigma_{22}}{\sigma_{e}}\right)^{2} \\
L_{12}=\frac{2}{3} E_{S}-\left(E_{S}-E_{T}\right) \frac{\sigma_{11} \sigma_{22}}{\left(\sigma_{e}\right)^{2}} ; \quad L_{S}=\frac{E_{S}}{3}+Q
\end{array}\right.
$$

where the component $Q$ is defined by the following relation:

$$
Q=\frac{1}{3} E_{S}\left[\left(\varepsilon_{11}-\varepsilon_{22}\right) \operatorname{coth}\left(\varepsilon_{11}-\varepsilon_{22}\right)-1\right]
$$

in which coth denotes the hyperbolic cotangent. 
It is worth noting that this form of the instantaneous modulus is different from that developed by Stören and Rice (1975); in the latter, the component $Q$ vanishes. This difference is the result of the difference between the two constitutive equations (12) and (13).

\subsubsection{Instantaneous modulus for the neo-Hookean model}

The instantaneous modulus in the case of the neo-Hookean model was given by Jia and Li (2013):

$$
\left\{\begin{array}{l}
L_{11}=2 \mu\left[e^{2 \varepsilon_{11}}+e^{-2\left(\varepsilon_{11}+\varepsilon_{22}\right)}\right] \quad ; \quad L_{22}=2 \mu\left[e^{2 \varepsilon_{22}}+e^{-2\left(\varepsilon_{11}+\varepsilon_{22}\right)}\right] \\
L_{12}=2 \mu e^{-2\left(\varepsilon_{11}+\varepsilon_{22}\right)} \quad ; \quad L_{S}=\frac{\mu}{2}\left[e^{2 \varepsilon_{11}}+e^{2 \varepsilon_{22}}\right]
\end{array}\right.
$$

\subsubsection{Rice's bifurcation criterion}

This criterion applies to a continuous medium undergoing a homogeneous strain state. Here, we consider a bilayer, as sketched in Fig. 2a, comprised of an upper metal layer $U$ and a lower elastomer layer $L$. This layer organization will be respected here and in the following. The initial thickness of the upper (resp. lower) layer is denoted by $h_{I}$ (resp. $H_{I}$ ). The strain localization is searched for as a bifurcation phenomenon, meaning that a non-homogeneous straining mode becomes possible (i.e., the uniqueness of the solution of the rate equations is lost). This non-homogeneity is considered as a planar localization band in the thickness (i.e., localized necking), defined by its normal $\vec{n}=(\cos \theta, \sin \theta)$ (see Fig. 2b). The velocity gradient inside and outside the band is denoted as $\underline{G}^{+}$and $\underline{G}^{-}$, respectively, while the corresponding nominal stresses are denoted as $\underline{N}^{+}$and $\underline{N}^{-}$.

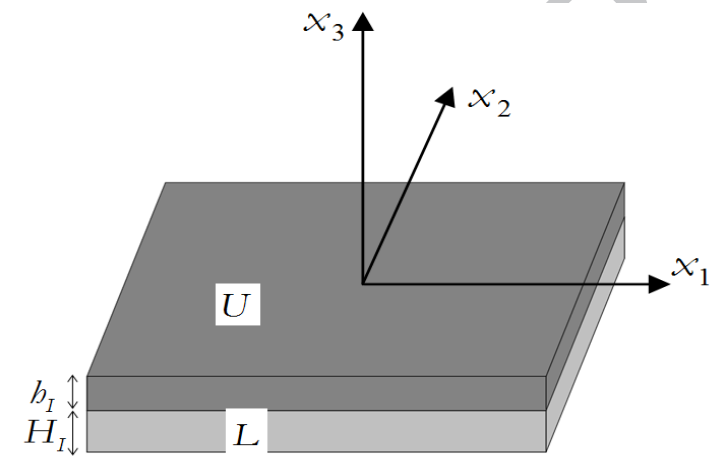

(a)

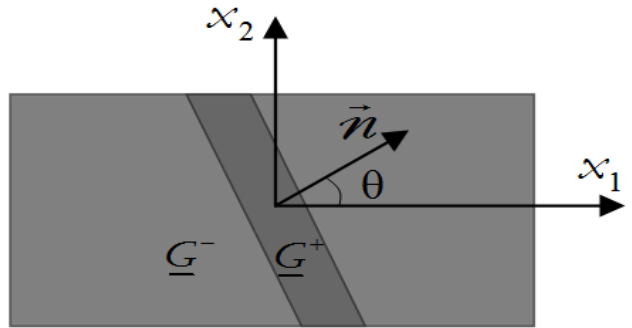

(b)

Fig. 2. Development of localized necking: (a) Schematic representation of the substrate-supported metal layer; (b) Orientation and shape of the localization band.

The two layers $U$ and $L$ are assumed to be perfectly adhered. Furthermore, they are submitted to a uniform pre-localization strain state with in-plane strains $\varepsilon_{11}, \varepsilon_{22}$ and $\varepsilon_{12}=0$.

With this adherence assumption and this specific loading, the velocity gradients $\underline{G}(U)$ and $\underline{G}(L)$ are equal to the overall velocity gradient $\underline{G}$, as indicated by the following equation:

$$
\underline{G}(U)=\underline{G}(L)=\underline{G}
$$


For each layer, the nominal stress rate $\underline{\dot{N}}$ is related to the velocity gradient $\underline{G}$ by:

$$
\underline{N}(U)=\mathscr{L}(U): \underline{G} \quad ; \quad \underline{N}(L)=\stackrel{\mathscr{L}}{=}(L): \underline{G}
$$

where $\stackrel{\mathscr{L}}{=}$ is an analytical tangent modulus, which is related to the instantaneous modulus $\underline{\underline{L}}$ by:

$$
\stackrel{\mathscr{L}}{=}=\underline{\underline{L}}+\underline{\underline{L}}_{1}-\underline{\underline{L}}_{2}-\underline{\underline{L}}_{3}
$$

Tensors $\underline{\underline{L}}_{1}, \underline{\underline{L}}_{2}$ and $\underline{\underline{L}}_{3}$ are given in the general three-dimensional case by the following expressions:

$$
L_{1 j k l l}=\sigma_{i j} \delta_{k l} \quad ; \quad L_{2 i j k l}=\frac{1}{2}\left[\delta_{i k} \sigma_{l j}+\delta_{i l} \sigma_{k j}\right] ; \quad L_{3 i j k l}=\frac{1}{2}\left[\sigma_{i k} \delta_{l j}-\sigma_{i l} \delta_{j k}\right] ; i, j, k, l=1,2,3
$$

where $\delta$ is the Kronecker delta. In the present case of plane-stress conditions, these tensors $\underline{\underline{L}}_{1}, \underline{\underline{L}}_{2}$ and $\underline{\underline{L}}_{3}$ will be adapted as usual, with the classical transformation into plane-stress formulation. In addition, tensor $\underline{\underline{L}}_{1}$ vanishes due to the incompressibility condition. More details on the determination of these tensors can be found in Abed-Meraim (2009), Haddag et al. (2009).

The continuity of the stress vector through the band of normal $\vec{n}$ is written as:

$$
\vec{n} \cdot \square h \underline{\dot{N}}(U)+H \underline{\dot{N}}(L) \boxminus=\overrightarrow{0}
$$

where $\square \underline{A} \square=\underline{A}^{+}-\underline{A}^{-}$designates the jump in a tensor quantity $\underline{A}$ across the chosen plane. Maxwell's compatibility condition, for the velocity field, states that there exists a vector $\vec{\beta}$ such that the jump in $\underline{G}$ reads:

$$
\square G \square=\vec{\beta} \otimes \vec{n}
$$

Combining Eqs. (25), (28) and (29), one obtains:

$$
[\vec{n} \cdot(b \stackrel{\varrho}{=}(U)+H \stackrel{\varrho}{=}(L)) \cdot \vec{n}] \cdot \vec{\beta}=\overrightarrow{0}
$$

This is a typical eigenvalue problem and the existence of a non-trivial solution for $\vec{\beta}$ (i.e., bifurcation condition $\vec{\beta} \neq 0$ ) requires that the following determinant vanishes:

$$
\operatorname{det}[\vec{n} \cdot(h \stackrel{\mathscr{L}}{=}(U)+H \stackrel{\mathscr{L}}{=}(L)) \cdot \overrightarrow{\boldsymbol{n}}]=0
$$

This latter equation gives a necessary condition for a localization band to appear. This condition of singularity for the acoustic tensor, denoted $\underline{C}$ and equal to $\vec{n}$. $(h \stackrel{\mathscr{L}}{=}(U)+H \stackrel{\mathscr{L}}{=}(L)) \cdot \vec{n}$, has also been shown to correspond to the loss of ellipticity of the rate equations that govern the associated boundary value problem. Equation (31) represents the extension to a bilayer of the bifurcation criterion introduced by Rice and coworkers in the case of a freestanding metal layer (see, e.g., Stören and Rice, 1975; Rice, 1976). 


\subsection{Imperfection approach}

In order to apply the $\mathrm{M}-\mathrm{K}$ approach, we add to the bilayer a groove in the form of a band on the upper metal layer (Fig. 3). To be consistent with various experimental observations, the band is assumed to initiate always in the upper metal layer.

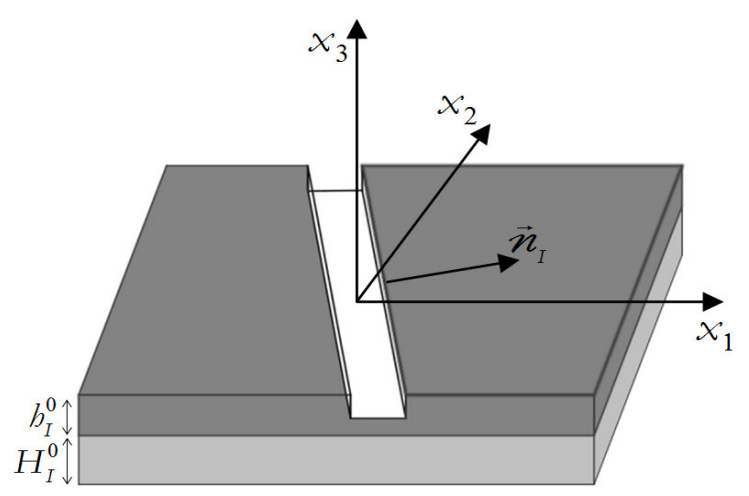

Fig. 3. M-K analysis of a bilayer (initial geometry and band orientation).

The following various thicknesses are introduced in order to establish the equations of the $\mathrm{M}-\mathrm{K}$ analysis:

- $h_{I}^{B}$ : initial thickness of layer $U$ inside the band (referred to as the defect zone).

- $h_{I}^{0}$ : initial thickness of layer $U$ outside the band (referred to as the uniform zone).

- $H_{I}^{B}$ : initial thickness of layer $L$ inside the band.

- $H_{I}^{0}$ : initial thickness of layer $L$ outside the band (equal to $H_{I}^{B}$ ).

On the basis of these notations, the initial size for the geometric imperfection (corresponding to the metal layer only), $\xi_{I}$, can be specified by:

$$
\xi_{I}=1-\frac{h_{I}^{B}}{h_{I}^{0}}
$$

As the adherence is perfect between layers $U$ and $L$, it is legitimate to assume the following equalities for the deformation gradients:

$$
\underline{F}^{B}(U)=\underline{F}^{B}(L)=\underline{F}^{B} \quad ; \quad \underline{F}^{0}(U)=\underline{F}^{0}(L)=\underline{F}^{0}
$$

In an Eulerian formulation, the perfect adherence is interpreted by the following equations:

$$
\underline{G}^{B}(U)=\underline{G}^{B}(L)=\underline{G}^{B} \quad ; \quad \underline{G}^{0}(U)=\underline{G}^{0}(L)=\underline{G}^{0}
$$

The $\mathrm{M}-\mathrm{K}$ analysis is based on three main equations:

- The kinematic compatibility condition between the band and the uniform zone (i.e., outside the band): this condition requires the displacement increments to be continuous across the band (Rice, 1976) and it is mathematically expressed (in a total Lagrangian form) by:

$$
\underline{F}^{B}=\underline{F}^{0}+\vec{c} \otimes \vec{n}_{I}
$$


The compatibility condition can also be expressed in a rate-type Eulerian formulation (equivalent to the Lagrangian form of Eq. (35)):

$$
\underline{G}^{B}=\underline{G}^{0}+\vec{\beta} \otimes \vec{n}
$$

where $\vec{n}_{I}$ and $\vec{n}$ refer to the initial and the current normal to the localization band, respectively.

- The equilibrium balance across the band interface: this balance is written in a total Lagrangian form as:

$$
\left(h_{I}^{B} \underline{B}^{B}(U)+H_{I}^{B} \underline{B}^{B}(L)\right) \cdot \vec{n}_{I}=\left(h_{I}^{0} \underline{B}^{0}(U)+H_{I}^{0} \underline{B}^{0}(L)\right) \cdot \vec{n}_{I}
$$

where $\underline{B}$ is the first Piola-Kirchhoff stress tensor related to the Cauchy stress tensor by:

$$
\underline{B}=J \underline{\sigma} \underline{F}^{-T}
$$

where $J$ is the Jacobian of the deformation gradient (equal to $\operatorname{det}(\underline{F})$ ). As the different materials used in this paper are assumed to be incompressible, $J$ is always equal to 1.

Using the Eulerian formulation, the equilibrium equation becomes:

$$
\left(h^{B} \underline{\sigma}^{B}(U)+H^{B} \underline{\sigma}^{B}(L)\right) \cdot \vec{n}=\left(h^{0} \underline{\sigma}^{0}(U)+H^{0} \underline{\sigma}^{0}(L)\right) \cdot \vec{n}
$$

- The constitutive equations (16) and (17).

\section{Numerical implementation and algorithmic aspects}

\subsection{Bifurcation analysis}

In order to predict the FLD, proportional strain paths are imposed to the bilayer:

$$
\frac{\dot{\varepsilon}_{22}}{\dot{\varepsilon}_{11}}=\rho
$$

The strain ratio $\rho$ is varied in the range $-1 / 2 \leq \rho \leq 1$ to span the complete FLD.

Taking Eq. (40) into account, it is possible to recast Eq. (24) (only the in-plane components pertaining to the pre-localization state are considered, the remaining components are deduced by the conditions of incompressibility and plane stress):

$$
\underline{G}(U)=\underline{G}(L)=\underline{G}=\left(\begin{array}{cc}
\dot{\varepsilon}_{11} & 0 \\
0 & \rho \dot{\varepsilon}_{11}
\end{array}\right)
$$

With this proportional straining, and considering that the metal layer is isotropic and follows the von Mises criterion, ratios $\sigma_{11} / \sigma_{e}$ and $\sigma_{22} / \sigma_{e}$ used in Eqs. (20) (for the flow theory) and (21) (for the deformation theory) are constant and given by:

$$
\frac{\sigma_{11}}{\sigma_{e}}=\frac{2+\rho}{\left[3\left(1+\rho+\rho^{2}\right)\right]^{1 / 2}} \quad ; \quad \frac{\sigma_{22}}{\sigma_{e}}=\frac{1+2 \rho}{\left[3\left(1+\rho+\rho^{2}\right)\right]^{1 / 2}}
$$


In this paper, the effective stress $\sigma_{e}$ is related to the effective strain $\varepsilon_{e}$ by the Hollomon law (Hollomon, 1945):

$$
\sigma_{e}=K \varepsilon_{e}^{N}
$$

where $K$ and $N$ are two material hardening parameters.

With this hardening law, the tangent and secant moduli $E_{T}$ and $E_{S}$, respectively, are given by:

$$
E_{T}=N K \varepsilon_{e}^{N-1} ; E_{S}=K \varepsilon_{e}^{N-1}
$$

where:

$$
\varepsilon_{e}=\frac{2\left(1+\rho+\rho^{2}\right)^{1 / 2}}{\sqrt{3}} \varepsilon_{11}
$$

Making use of Eqs. (40)-(45), the instantaneous moduli (20), (21) and (23) for the different materials can be expressed as functions that only depend on the material parameters, the strain ratio $\rho$ and $\varepsilon_{11}$. Consequently, for a given bilayer (when the materiel parameters of the different layers and the initial thicknesses $h_{I}$ and $H_{I}$ are fixed), the acoustic tensor $\underline{C}$ becomes a function of $\rho$, the band orientation $\theta$ and $\varepsilon_{11}$.

The prediction of the entire FLD of the bilayer is based on two nested loops:

- For $\rho=-1 / 2$ to $\rho=1$ at user-defined intervals (here, we take intervals of 0.1 )

○ For $\theta=0^{\circ}$ to $\theta=90^{\circ}$ at user-defined intervals (here, we take intervals of $1^{\circ}$ )

- Solve the highly non-linear Eq. (31) with respect to the unique unknown $\varepsilon_{11}$. The solution of Eq. (31) is called the critical strain $\varepsilon_{11}^{*}$ corresponding to $\rho$ and $\theta$.

The smallest critical strain $\varepsilon_{11}^{*}$ over all angles $\theta$ and the corresponding angle define, respectively, the localization limit strain $\varepsilon_{11}^{L}$ and the necking band orientation for the current strain ratio $\rho$.

\section{2. $M-K$ analysis}

Unlike the bifurcation analysis, the solution method for the $\mathrm{M}-\mathrm{K}$ analysis depends on whether flow theory or deformation theory is applied to model the mechanical behavior of the metal layer. In the case of Metal (flow theory)/Elastomer (neo-Hookean) combination, an incremental solution is required. However, in the case of Metal (deformation theory)/Elastomer (neo-Hookean) combination, a direct solution can be given without using an incremental procedure. To be clear, in the following developments, the two cases will be treated separately.

\subsubsection{Metal (deformation theory)/Elastomer (neo-Hookean)}

The in-plane normal components of the deformation gradient outside the band are prescribed as follows:

$$
F_{11}^{0}=e^{\varepsilon_{11}^{0}} \quad ; \quad F_{22}^{0}=e^{\varepsilon_{22}^{0}}=e^{\rho \varepsilon_{11}^{0}}
$$


with the remaining in-plane components set to be:

$$
F_{12}^{0}=F_{21}^{0}=0
$$

Substituting the components of $\underline{F}^{0}$ (Eqs. (46) and (47)) into Eq. (35) gives the in-plane components of the deformation gradient in the band $\underline{F}^{B}$ :

$$
\underline{F}^{B}=\left[\begin{array}{cc}
e^{\varepsilon_{11}^{0}}+C_{1} n_{I 1} & C_{1} \boldsymbol{n}_{I 2} \\
C_{2} \boldsymbol{n}_{I 1} & e^{\rho \varepsilon_{11}^{0}}+C_{2} \boldsymbol{n}_{I 2}
\end{array}\right]
$$

In view of Eq. (48), we can easily conclude that $\underline{F}^{B}$ depends on $\rho, \varepsilon_{11}^{0}, \vec{c}$ and $\vec{n}_{I}$ (or equivalently the initial orientation $\theta_{I}$ ).

The first Piola-Kirchhoff stress tensors $\underline{B}^{B}(U), \underline{B}^{B}(L), \underline{B}^{0}(U), \underline{B}^{0}(L)$ can be expressed through Eq. (38):

$$
\begin{array}{ll}
\underline{B}^{B}(U)=\underline{\sigma}^{B}(U)\left[\underline{F}^{B}\right]^{-T} \quad ; \quad \underline{B}^{B}(L)=\underline{\sigma}^{B}(L)\left[\underline{F}^{B}\right]^{-T} \\
\underline{B}^{0}(U)=\underline{\sigma}^{0}(U)\left[\underline{F}^{0}\right]^{-T} \quad ; \quad \underline{B}^{0}(L)=\underline{\sigma}^{0}(L)\left[\underline{F}^{0}\right]^{-T}
\end{array}
$$

$\underline{\sigma}^{B}(U)$ and $\underline{\sigma}^{B}(L)$ (resp., $\left.\underline{\sigma}^{0}(U), \underline{\sigma}^{0}(L)\right)$ are expressed through Eqs. (16), (17) $)_{2}$ and $(17)_{3}$ as functions of $\underline{V}^{B}$ (resp., $\left.\underline{V}^{0}\right)$, which in turn are functions of $\underline{F}^{B}$ (resp., $\left.\underline{F}^{0}\right)$. Therefore, $\underline{B}^{B}(U), \underline{B}^{B}(L), \underline{B}^{0}(U)$, $\underline{B}^{0}(L)$ are functions of $\rho, \varepsilon_{11}^{0}, \vec{c}$ and $\vec{n}_{I}$.

Knowing the material parameters and the initial thicknesses of the two layers $U$ and $L$ inside and outside the band, the $\mathrm{M}-\mathrm{K}$ problem is reduced to the solution of the equilibrium balance (37) (two scalar equations). This equilibrium balance is function of $\rho, \varepsilon_{11}^{0}, \vec{c}$ and $\vec{n}_{I}$. For a given value of the strain-path parameter $\rho$ and initial band orientation $\theta_{I}$ (i.e., $\vec{n}_{I}$ ), Eq. (37) becomes function of the only independent unknowns $\varepsilon_{11}^{0}$ and $\vec{C}$. Therefore, we have two scalar equations for three unknowns ( $\varepsilon_{11}^{0}$ and the two components of $\vec{c}$ ). In order to solve completely this problem, it is necessary to add a supplementary or a "matching" equation or numerical constraint.

As will be demonstrated later, plastic strain localization is said to occur when the straining stops outside the band (unloading), while the band continues to undergo plastic loading. Accordingly, the strain component $\varepsilon_{11}^{0}$ and the equivalent deformation $\varepsilon_{e q}^{0}$ outside the band reach their maximum at the onset of unloading. We therefore refer to this limiting value as the critical strain for localized necking, as it represents the state where the deformation becomes concentrated in the band while the reminder of the sheet begins to unload. This criterion, which in general is equivalent to $d \varepsilon_{11}^{0} / d \varepsilon_{11}^{B}=0$ or $d \varepsilon_{e q}^{0} / d \varepsilon_{e q}^{B}=0$, is used here as indicator of critical strain.

Consequently, when the material parameters and initial layer thicknesses are known and when the strain ratio $\rho$ and initial orientation $\theta_{I}$ are fixed, the $\mathrm{M}-\mathrm{K}$ problem can be posed as a maximization 
problem with constraint: maximize $\varepsilon_{11}^{0}$ subject to the two scalar equilibrium equations (37). This maximization problem admits always a unique solution, which allows us to determine $\varepsilon_{11}^{0}$ and $\vec{c}$, and hence to solve completely the M-K problem.

Similarly to the case of bifurcation analysis, the prediction of the entire FLD of the bilayer is based on two nested loops:

- For $\rho=-1 / 2$ to $\rho=1$ at user-defined intervals (here, we take intervals of 0.1 )

○ For $\theta_{I}=0^{\circ}$ to $\theta_{I}=90^{\circ}$ at user-defined intervals (here, we take intervals of $1^{\circ}$ )

- Search for the critical strain $\varepsilon_{11}^{*}$ as a solution of the following maximization problem with constraint:

$$
\text { Maximize } \varepsilon_{11}^{0} \text { subject to }\left[\left(b_{I}^{B} \underline{B}^{B}(U)+H_{I}^{B} \underline{B}^{B}(L)\right)-\left(b_{I}^{0} \underline{B}^{0}(U)+H_{I}^{0} \underline{B}^{0}(L)\right)\right] \cdot \vec{n}_{I}=\overrightarrow{0}
$$

The smallest critical strain $\varepsilon_{11}^{*}$, solution of the above optimization problem, over all initial angles $\theta_{I}$ and the corresponding current angle define, respectively, the localization limit strain $\varepsilon_{11}^{L}$ and the necking band orientation for the current strain ratio $\rho$.

\subsubsection{Metal (flow theory)/Elastomer (neo-Hookean)}

In these conditions and due to the rate-type formulation of the flow theory, the Eulerian form (summarized by Eqs. (36), (39) and the constitutive equations of the metal (17) $)_{1}$ and of the elastomer $(17)_{3}$ ) of the $\mathrm{M}-\mathrm{K}$ equations is more suitable than the Lagrangian form. These equations shall be verified at each time increment. In practice, the $\mathrm{M}-\mathrm{K}$ problem must be solved in an incremental manner. Before defining the incremental algorithm used, and for the sake of clarity, it is necessary to define the prescribed quantity used in each increment.

\subsubsection{Choice of the prescribed quantity}

In the majority of works carried out for incrementally solving the $\mathrm{M}-\mathrm{K}$ problem, the equivalent strain rate $\dot{\varepsilon}_{e q}^{0}$ or a component of the strain rate (generally $\dot{\varepsilon}_{11}^{0}$ ) outside the band is imposed as the prescribed quantity. However, the corresponding strain quantities $\left(\varepsilon_{e q}^{0}, \varepsilon_{11}^{0}\right)$ increase monotonically before strain localization, while they remain constant (in the case of rigid-plasticity, which is the case in this subsection) or decrease (in the case of elasto-plastic flow theory or deformation theory) after strain localization. On the contrary, strain quantities inside the band $\left(\varepsilon_{e q}^{B}, \varepsilon_{11}^{B}, \varepsilon_{33}^{B} \ldots\right)$ increase monotonically both before and after strain localization. Hence, it is more suitable to use one of these strain rates inside the band as prescribed quantity instead of strain rates outside the band. This choice permits to carry out stable computations and avoid several numerical difficulties. In our case, $\dot{\varepsilon}_{33}^{B}$ is used as the prescribed quantity. Because the constitutive framework is assumed to be strain-rate insensitive, $\dot{\varepsilon}_{33}^{B}$ may be chosen quite freely. For convenience, it is assumed to be constant and equal to -1 , from which it follows that $\left|\varepsilon_{33}^{B}\right|$ is numerically equal to time $t$. Combining this choice with the incompressibility 
condition $G_{11}^{B}+G_{22}^{B}+G_{33}^{B}=0$, we can obtain the following form for the velocity gradient in the band $\underline{G}^{B}$ (in-plane components only due to plane-stress conditions):

$$
\underline{G}^{B}=\left[\begin{array}{cc}
\dot{\varepsilon}_{11}^{B} & G_{12}^{B} \\
G_{21}^{B} & 1-\dot{\varepsilon}_{11}^{B}
\end{array}\right]
$$

On the other hand, the deformation in the uniform zone is assumed to be such that:

$$
\frac{G_{22}^{0}}{G_{11}^{0}}=\frac{\dot{\varepsilon}_{22}^{0}}{\dot{\varepsilon}_{11}^{0}}=\rho
$$

It is further assumed that the $x_{1}$ and $x_{2}$-directions are the in-plane principal strain directions in the uniform zone, by which it is understood that:

$$
G_{12}^{0}=G_{21}^{0}=0
$$

Making use of the compatibility condition (36) together with Eqs. (51), (52) and (53), we can obtain that:

$$
\left\{\begin{array}{l}
\dot{\varepsilon}_{11}^{B}=\dot{\varepsilon}_{11}^{0}+\beta_{1} n_{1} \\
G_{12}^{B}=\beta_{1} n_{2} \\
G_{21}^{B}=\beta_{2} n_{1} \\
\dot{\varepsilon}_{22}^{B}=1-\dot{\varepsilon}_{11}^{B}=\rho \dot{\varepsilon}_{11}^{0}+\beta_{2} n_{2}
\end{array}\right.
$$

By combining Eqs. (54) $)_{1}$ and $(54)_{4}, \dot{\varepsilon}_{11}^{0}$ can be obtained:

$$
\dot{\varepsilon}_{11}^{0}=\frac{1-\beta_{1} n_{1}-\beta_{2} n_{2}}{1+\rho}
$$

The current normal to the band $\vec{n}$ is equal to $(\cos \theta, \sin \theta)$ and its orientation $\theta$ is related, under the condition of Eq. (53), to the initial band orientation $\theta_{I}$ by the expression:

$$
\tan \theta=\exp \left[(1-\rho) \varepsilon_{11}^{0}\right] \tan \theta_{I}
$$

Analyzing Eqs. (51)-(56), it is clear that it is sufficient to know the scalars $\beta_{1}$ and $\beta_{2}$ to determine completely $\underline{G}^{0}$ and $\underline{G}^{B}$. Indeed, if $\beta_{1}$ and $\beta_{2}$ are known, Eq. (55) combined with Eq. (56) becomes a non-linear first-order differential equation for the unknown $\varepsilon_{11}^{0}$. Solving this equation permits to determine $\varepsilon_{11}^{0}, \dot{\varepsilon}_{11}^{0}$ and then the other components of the velocity gradients $\underline{G}^{0}$ and $\underline{G}^{B}$ through Eq. (54).

\subsubsection{Incremental algorithm}

When the input parameters are known, an implicit incremental algorithm is constructed to determine the major strain $\varepsilon_{11}^{0}$ for each strain ratio $\rho$ and each initial orientation $\theta_{I}$. This algorithm is detailed in Appendix A.

The general algorithm used to predict the FLD of the bilayer is based on three nested loops: 
- For $\rho=-1 / 2$ to $\rho=1$ at user-defined intervals (here, we take intervals of 0.1 )

○ For $\theta_{I}=0^{\circ}$ to $\theta_{I}=90^{\circ}$ at user-defined intervals (here, we take intervals of $1^{\circ}$ )

- Apply the implicit incremental algorithm (as described in Appendix A) to calculate the major strain $\varepsilon_{11}^{0}$. The computation is stopped when the necking criterion (A.3) is satisfied and the corresponding strain $\varepsilon_{11}^{0}$ is considered to be the critical strain $\varepsilon_{11}^{*}$.

The smallest critical strain $\varepsilon_{11}^{*}$ solution of the previous problem over all initial angles $\theta_{I}$ and the corresponding current angle define, respectively, the localization limit strain $\varepsilon_{11}^{L}$ and the necking band orientation for the current strain ratio $\rho$.

\section{Results and discussions}

This section is divided into two main subsections: the freestanding metal layer results, where we revisit some well-known results from the literature (Hutchinson and Neale, 1978b; Jia and Li, 2013), and the bilayer results, which represent the main novelty of this paper.

\subsection{Freestanding metal layer}

In this subsection, two values for the hardening exponent are used: $N=0.22$ (typical of steels and aluminum alloys) and $N=0.5$ (more representative of brass materials). Both bifurcation results and $\mathrm{M}-\mathrm{K}$ predictions are insensitive to the value of the hardening parameter $K$. For this reason, its numerical value is not mentioned in this subsection.

\subsubsection{Bifurcation analysis predictions}

Comparisons between our numerical predictions and the reference results reported in Hutchinson and Neale (1978b) are shown in Fig. 4. The solid and dashed lines represent the results given by our numerical tool, while the dotted graphs with symbols $(\Delta, \boldsymbol{\Lambda}, \boldsymbol{\square})$ correspond to those published in Hutchinson and Neale (1978b). It is clear that both sets of FLD results match perfectly, which provides at least a partial validation of our algorithm and of the accuracy of the developed model. Here, the two versions of the deformation theory are used: the hyperelastic version developed by Hutchinson and Neale (1978b), referred to as H-N, and the hypoelastic version developed by Stören and Rice (1975), referred to as S-R. In the positive biaxial stretching range, it is found that the principal axes of strain do not rotate with the associated bifurcation mode and, accordingly, the component $L_{S}$ that enters the expression of the instantaneous modulus $\underline{\underline{L}}$ (see Eqs. (19) and (21)) does not affect the bifurcation governing equations (30). This implies that this component $L_{S}$ does not play any role in the expression of the bifurcation criterion (31) neither (in the range of positive biaxial stretching) and, accordingly, the results of Hutchinson and Neale (1978b) and Stören and Rice (1975) are identical as expected (see the right-hand sides of Fig. 4a and b). As demonstrated in Hill (1952), bifurcation cannot occur in the range of positive minor strain $\left(\varepsilon_{2}>0\right)$ when the rigid-plastic flow theory is used; therefore, only the available left-hand side of the FLD is presented in Fig. 4a and b. 
For $\rho<0$, various band orientations $\theta$ were considered to determine the angle that corresponds to the minimum limit strain. In the biaxial tension range $(\rho \geq 0), \theta=0$ is the minimizing band orientation. For this reason the localization band angle $\theta$ is presented only in the negative $\rho$ range. Unlike the FLD predictions, some discrepancies are observed when we compare the localization band orientations predicted by our tool (see Fig. 4c and d) with the predictions reported in Hutchinson and Neale (1978b), especially for the case of rigid-plastic flow theory. Because in the case of rigid-plastic flow theory the localization band orientation is given by the following analytical formula (see Hill's (1952) analysis):

$$
\theta=\arctan (\sqrt{-\rho})
$$

it is then easy to verify that our results follow exactly this analytical formula, which validates our numerical predictions.

In the case of the deformation theory, where the observed discrepancies are smaller and correspond especially to the band orientations associated with small values of $\rho$, we also verified the validity of our predictions by comparison with the recent results reported in Jia and Li (2013).

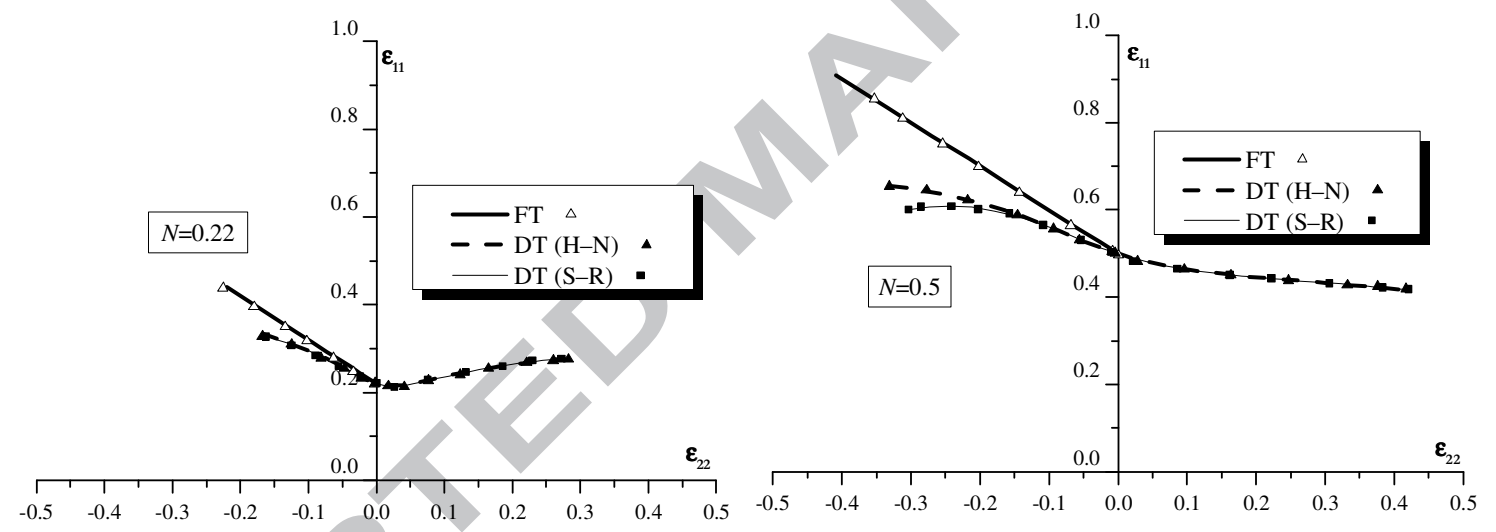

(a)

(b)

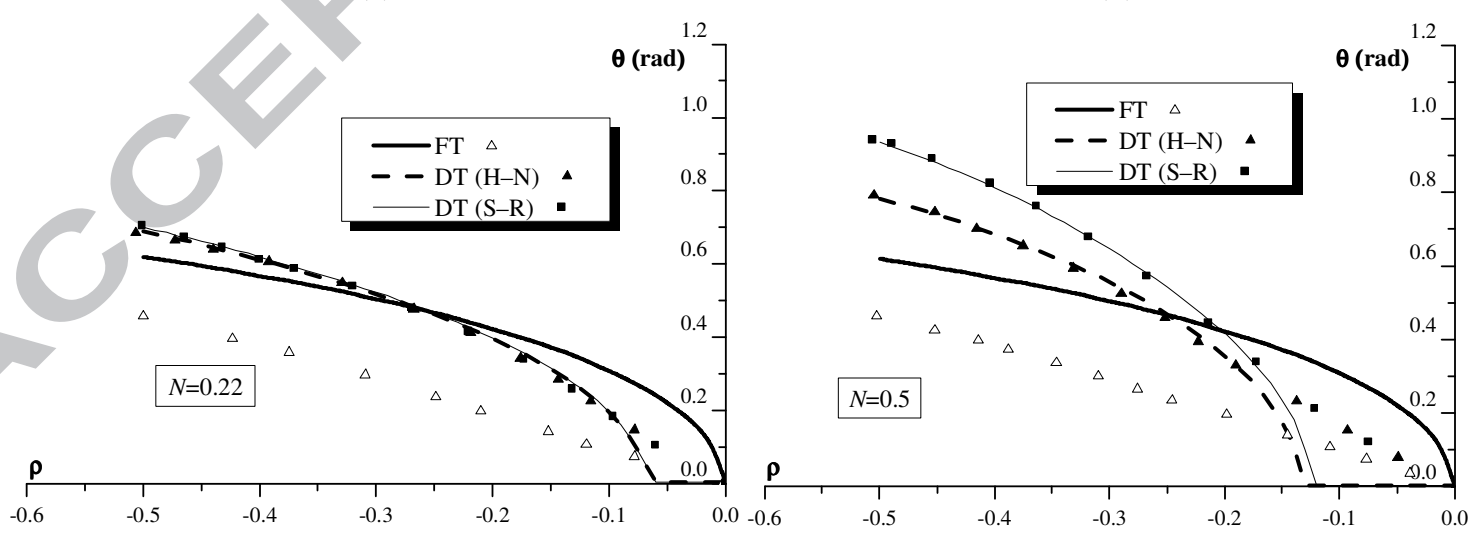

(c)

(d)

Fig. 4. FLDs and localization band orientations for a freestanding metal layer, as predicted by the bifurcation theory; comparison of flow theory with two versions of deformation theory: (a) FLDs 


$$
\begin{aligned}
& (N=0.22) \text {; (b) FLDs }(N=0.5) \text {; (c) Localization band orientation } \theta(N=0.22) \text {; (d) Localization } \\
& \text { band orientation } \theta(N=0.5) .
\end{aligned}
$$

\subsubsection{M-K analysis predictions}

In order to emphasize the effects of geometric imperfections on localized necking, we plot in Fig. 5 the evolution of the opposite of the thickness strain outside the band $-\varepsilon_{33}^{0}$ as a function of its counterpart inside the band $-\varepsilon_{33}^{B}$. This evolution can be used as a reliable indicator for the onset of localized necking. Indeed, the current size $\xi$ for the geometric imperfection can be expressed as a function of $\xi_{I}, \varepsilon_{33}^{B}$ and $\varepsilon_{33}^{0}$ by:

$$
\xi=1-\left(1-\xi_{I}\right) \exp \left(\varepsilon_{33}^{B}-\varepsilon_{33}^{0}\right)
$$

The hardening exponent $N$ is taken equal to 0.22 . The evolution of $-\varepsilon_{33}^{0}$ is plotted for four strain ratios: $\rho=-0.5$ (uniaxial tension), $\rho=0$ (plane strain tension), $\rho=0.5$ (biaxial tension), and $\rho=1$ (equibiaxial tension).

The initial band orientation is taken to be equal to $0.2 \mathrm{rad}$ for the first strain ratio and $0 \mathrm{rad}$ for the other strain ratios. The dot reported on each curve indicates the onset of strain localization. As demonstrated in Fig. 5, the critical strain $\varepsilon_{11}^{*}$ is equal to the maximum value of the major principal strain attained outside the band (which is considered previously as a definition of critical strain). It should be clarified that the localization strains given by Fig. 5, for different values of strain ratio $\rho$ and different values of initial band orientation $\theta_{I}$, do not all correspond to localization limit strains. This is only the case for the strain ratios $\rho=0, \rho=0.5$ and $\rho=1$, for which the initial band orientation taken (i.e., $\theta_{I}=0$ ) is that minimizing the critical strains $\varepsilon_{11}^{*}$. For $\rho=-0.5$, the localization limit strain will be obtained by minimizing the critical strains $\varepsilon_{11}^{*}$ over all initial band orientations $\theta_{I}$. Another observation from Fig. 5 is that the two plasticity theories exhibit quite different trends in the post-localization domain. In the case of deformation theory, after the onset of localization the material inside the band continues to deform plastically in a monotonous manner ( $-\varepsilon_{33}^{B}$ increases), while the material outside the band undergoes unloading ( $-\varepsilon_{33}^{0}$ decreases). In fact, as the deformation theory is not an acceptable plasticity theory in the case of unloading, it cannot be used for post-localization analysis. Accordingly, the post-localization regime in the case of deformation theory is represented in Fig. 5 by dashed lines for indication purposes only. Indeed, these dashed curves, which are extrapolated beyond their appropriate limit, should only be regarded as schematic, and do not have a sound theoretical basis. In the case of flow theory, however, because elasticity is not considered, the strain $-\varepsilon_{33}^{0}$ remains constant in the post-localization domain, while $-\varepsilon_{33}^{B}$ increases monotonically as previously.

Fig. 5 also reveals that the flow theory is more sensitive to the initial imperfection than the deformation theory, especially in the biaxial loading range. In both plasticity theories, the imperfection 
has a destabilizing role that precipitates the occurrence of strain localization and, certainly, when the problem is analyzed on the basis of flow theory, localization cannot be predicted for positive strain ratios without the introduction of imperfection.

The ratio $\varepsilon_{33}^{B} / \varepsilon_{33}^{0}$ at the localization point decreases with decreasing initial imperfection. Namely, when an imperfection is small, intensive strain localization starts suddenly, although the strain inside the band is comparable in magnitude to that outside the band.

Another noteworthy observation is that in the pre-localization regime and until the onset of localization, the two plasticity models behave identically when the strain ratio is equal to 0 (plane strain tension), regardless of the value of the initial imperfection $\xi_{I}$. For the other values of $\rho$, the deformation theory always predicts earlier strain localization when compared to flow theory. These observations are also confirmed by the FLDs reported in Fig. 6.

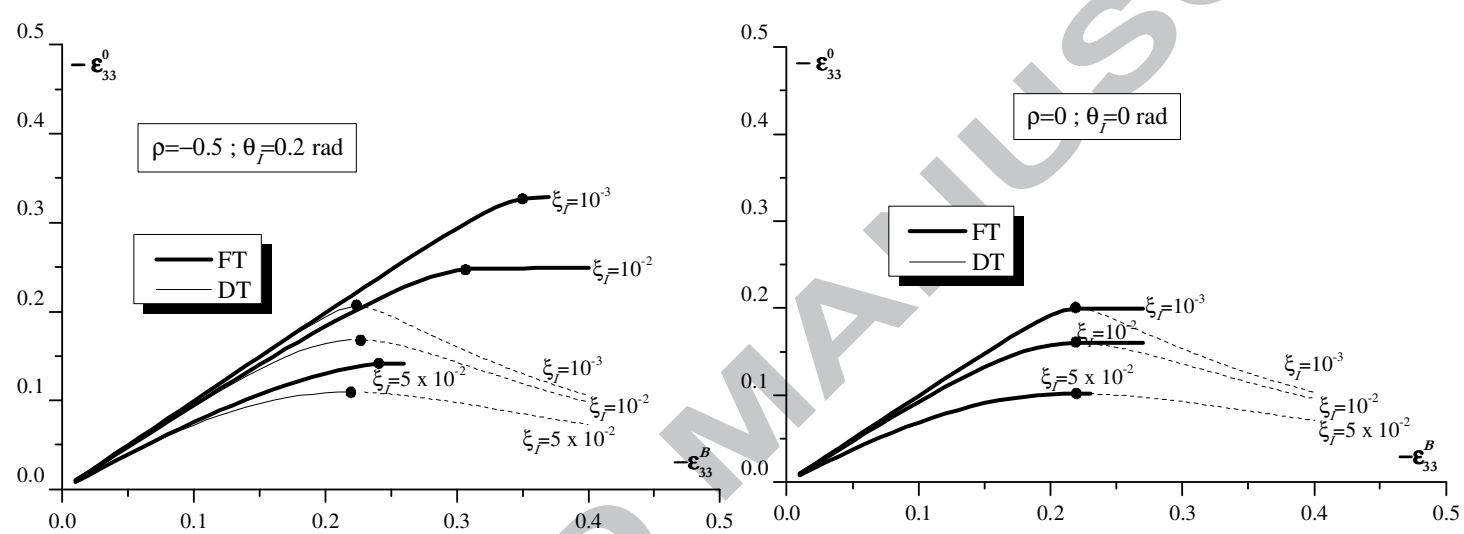

(a)

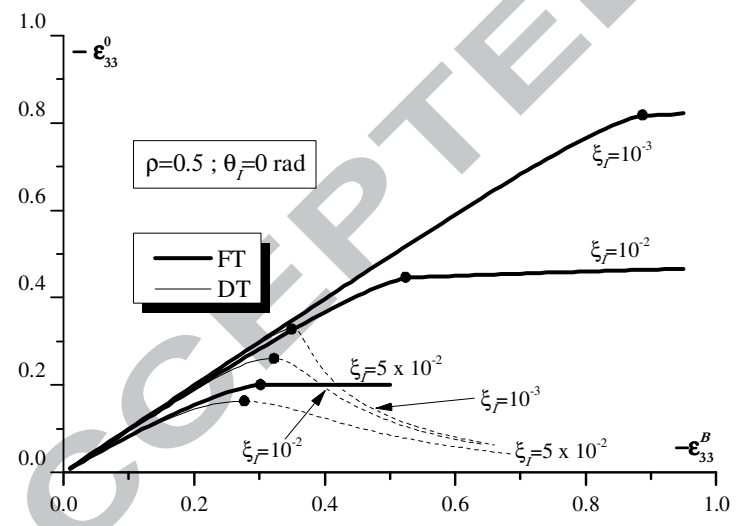

(c) (b)

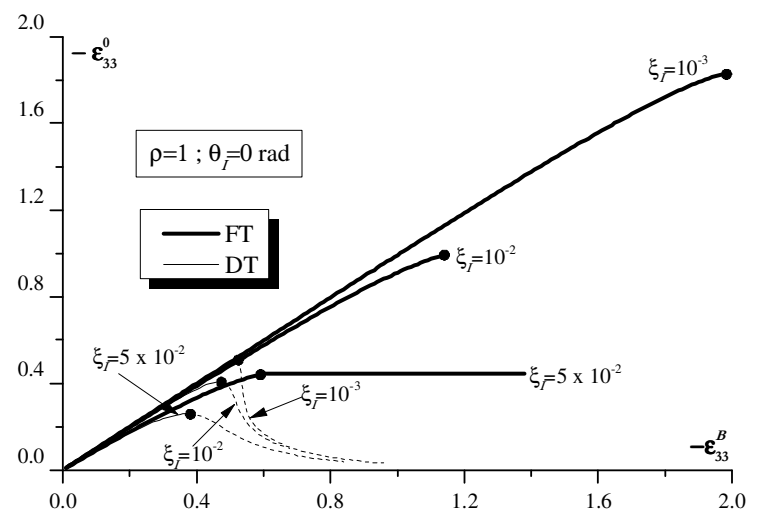

(d)

Fig. 5. Evolution of $-\varepsilon_{33}^{0}$ as a function of $-\varepsilon_{33}^{B}$ for a freestanding metal layer, as predicted by the $\mathrm{M}-$ $\mathrm{K}$ approach; comparison of flow theory with deformation theory ( $N=0.22$ ): (a) $\rho=-0.5$ and $\theta_{I}=0.2 \mathrm{rad}$; (b) $\rho=0$ and $\theta_{I}=0 \mathrm{rad}$; (c) $\rho=0.5$ and $\theta_{I}=0 \mathrm{rad}$; (d) $\rho=1$ and $\theta_{I}=0 \mathrm{rad}$.

The effect of the initial geometric imperfection $\xi_{I}$ on the shape and the location of the FLDs is investigated in Fig. 6. The analysis compares the predictions of both plasticity theories for two values 
of strain hardening exponent ( $N=0.22$ and $N=0.5$ ). In view of these results, some conclusions can be drawn:

- For both plasticity theories, the limit strains given by the bifurcation analysis set an upper bound to those yielded by the M-K approach. Indeed, Fig. 6 demonstrates that the FLDs predicted by the M-K analysis tend towards the FLD predicted by bifurcation analysis when $\xi_{I}$ tends to zero. In other words, the effect of an initial imperfection is essentially to shift the FLD downwards. This observation is natural considering the similarity of the mathematical formulations of the two approaches (M-K and bifurcation): if the amount of initial imperfection is set equal to 0 in the imperfection model, the problem is reduced to the bifurcation analysis.

- The limit strains given by the deformation theory coincide with those predicted by the flow theory for plane strain tension $(\rho=0)$. This result is always true for both localization approaches (bifurcation theory and $\mathrm{M}-\mathrm{K}$ analysis) and is consistent with Fig. 5 and with the results reported in Hutchinson and Neale (1978b).

- For all of the other strain paths $(\rho \neq 0)$, the limit strains predicted by the deformation theory are found to locate at realistic strain levels, and are always lower than their counterparts predicted by the flow theory. This result is also true for both localization approaches (bifurcation theory and $\mathrm{M}-\mathrm{K}$ analysis) and is consistent with Fig. 5.

- Despite the sound fundamental basis of the flow theory, its predictions seem to be overly sensitive to initial imperfections and are also relatively high, especially in the biaxial stretching range $(\rho>0)$. This observation is attributable to the infinite limit strains given by bifurcation analysis coupled with flow theory for $\rho>0$.

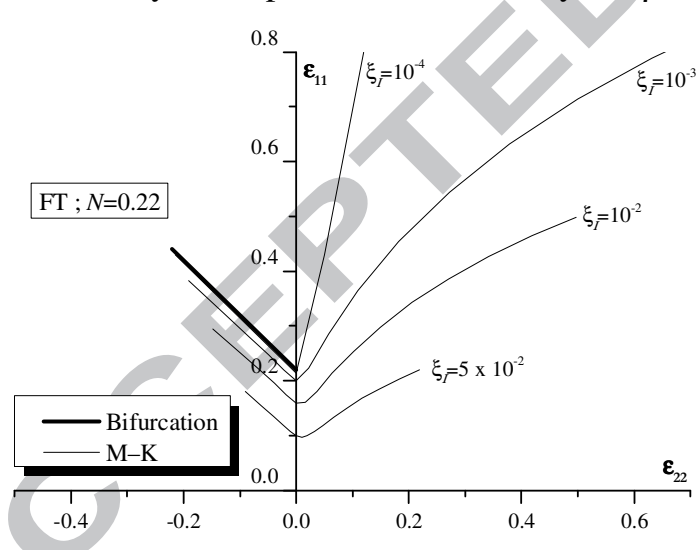

(a)

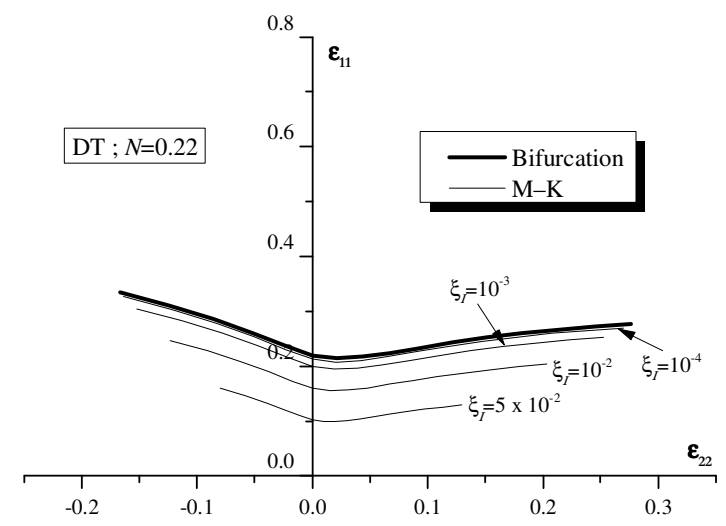

(b) 


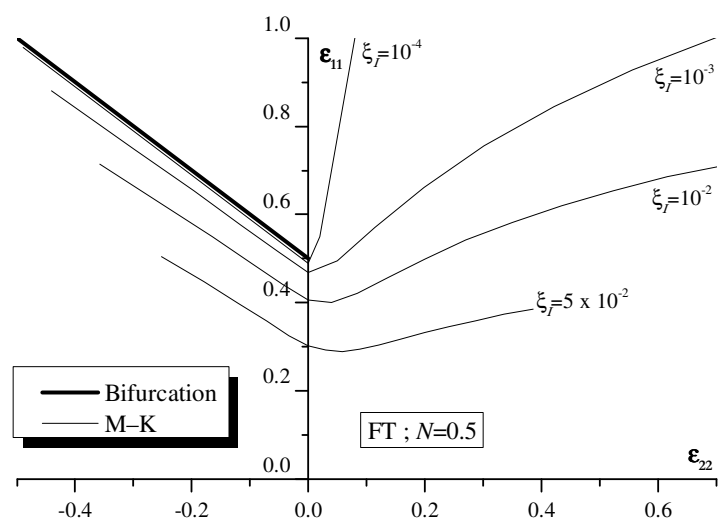

(c)

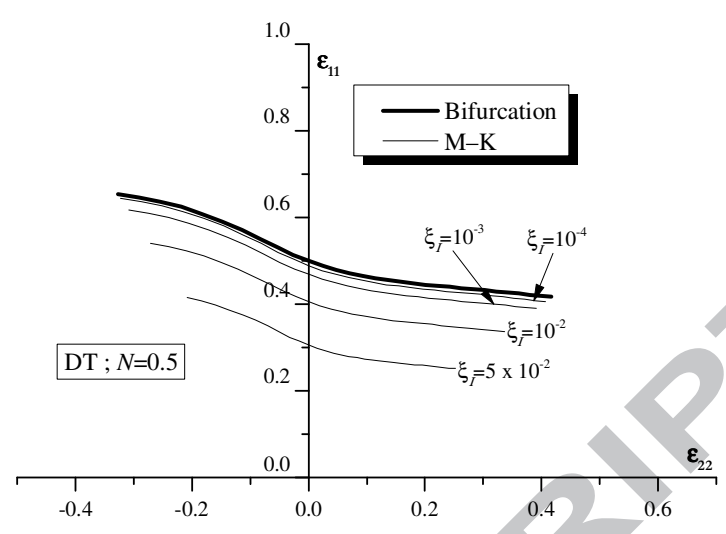

(d)

Fig. 6. Effect of $\xi_{I}$ on the shape and the location of the FLDs for a freestanding metal layer; comparison of flow theory with deformation theory: (a) Flow theory ( $N=0.22$ ); (b) Deformation theory ( $N=0.22)$; (c) Flow theory $(N=0.5)$; (d) Deformation theory $(N=0.5)$.

As reflected by Figs. 5 and 6, the introduction of an initial geometric imperfection leads to an important reduction of the critical strains (especially when flow theory is used). To further illustrate this feature, Fig. 7 shows the evolution of the limit strain $\varepsilon_{11}^{L}$ as a function of $\xi_{I}$ for a hardening exponent $N=0.22$ and four strain paths: $\rho=-0.5, \rho=0, \rho=0.5$ and $\rho=1$. As revealed, the limit strain $\varepsilon_{11}^{L}$ depends strongly on the initial imperfection $\xi_{I}$, especially for the case of positive strain ratios $(\rho=0.5$ and $\rho=1)$ and when the flow theory is used. In this specific case, localization may be expected to occur at reasonable values of $\varepsilon_{11}^{L}$ only when the amount of initial imperfection is sufficiently large. Note also that Fig. 7 confirms once again that the two plasticity theories predict the same limit strains in the case of plane strain tension $(\rho=0)$, irrespective of whether imperfections are included or not.

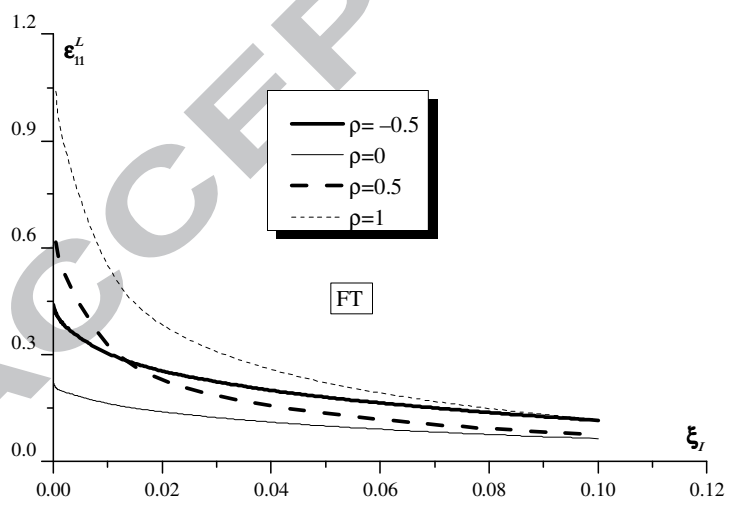

(a)

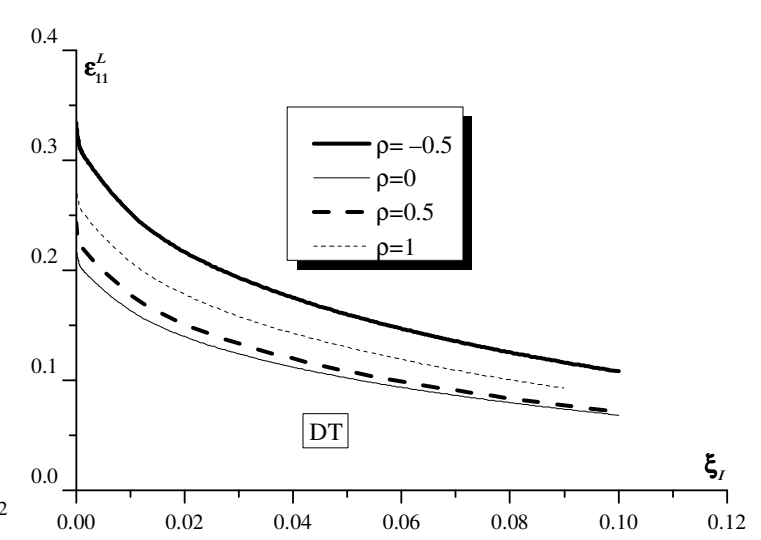

(b)

Fig. 7. Evolution of the limit strain $\varepsilon_{11}^{L}$ with the initial imperfection $\xi_{I}$ for a freestanding metal layer and for four different strain paths; comparison of flow theory with deformation theory ( $N=0.22$ ): (a) Flow theory; (b) Deformation theory. 
The dependence of the critical strain $\varepsilon_{11}^{*}$ on both the initial band orientation $\theta_{I}$ and the current band orientation $\theta$ is illustrated by the curves in Fig. 8. The strain ratio $\rho$ is taken here equal to -0.5 and the hardening exponent $N$ is taken equal to 0.22 . In these figures, both plasticity theories are investigated in which the bifurcation results are compared with the predictions of the $\mathrm{M}-\mathrm{K}$ analysis for three values of $\xi_{I}\left(10^{-3}, 10^{-2}, 5 \times 10^{-2}\right)$. The minimum critical strain $\varepsilon_{11}^{*}$ over all orientations gives the limit strain $\varepsilon_{11}^{L}$ at which the inception of localization is first possible.

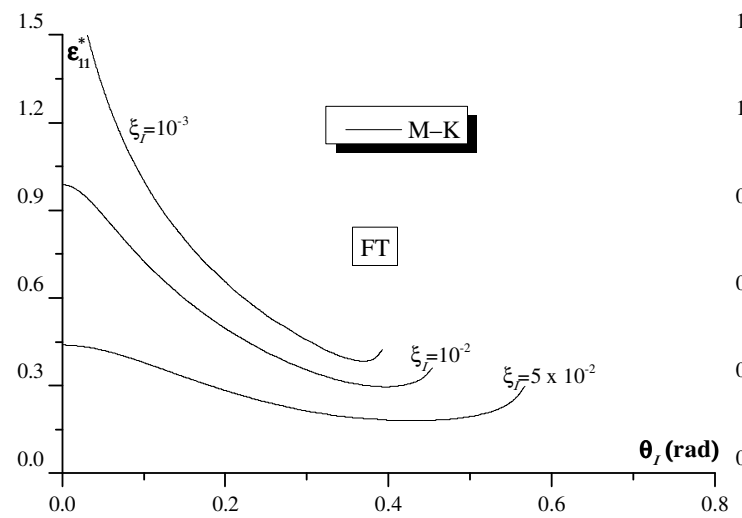

(a)

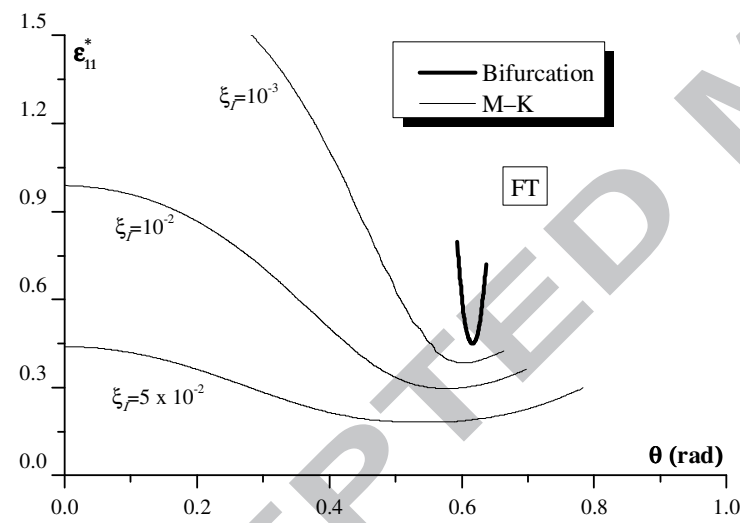

(c)

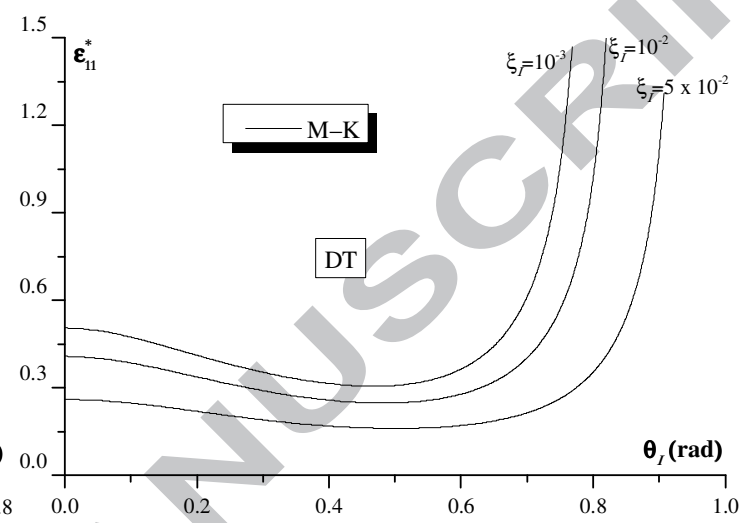

(b)

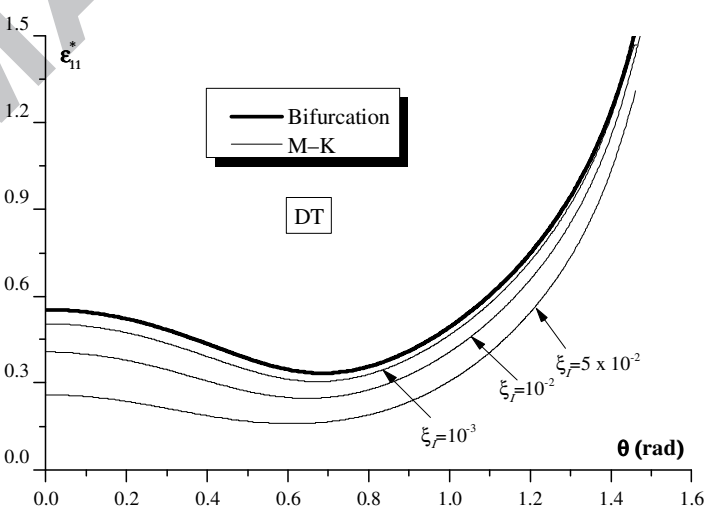

(d)

Fig. 8. Evolution of the critical strain $\varepsilon_{11}^{*}$ as a function of necking band orientation for a freestanding metal layer; comparison of flow theory with deformation theory $(\rho=-0.5, N=0.22$ ): (a) Initial orientation with flow theory; (b) Initial orientation with deformation theory; (c) Current orientation with flow theory; (d) Current orientation with deformation theory.

\subsection{Metal/Elastomer bilayer}

In this subsection, two bilayer combinations are considered; namely, a metal layer (modeled with flow theory) supported by an elastomer substrate and a metal layer (modeled with deformation theory) supported by an elastomer substrate. In what follows, a shorthand notation for the first (resp. second) combination will be designated as FT/NH (resp. DT/NH). In all of the calculations reported in this subsection, the hardening parameters of the metal layer are taken as $K=1000 \mathrm{MPa}$ and $N=0.22$. 
The shear modulus of the elastomer layer is fixed to $22 \mathrm{MPa}$. This latter choice is based on data for polyurea (Amirkhizi et al., 2006).

\subsubsection{Bifurcation analysis results}

Fig. 9 reflects the effect of the elastomer layer and its initial thickness $H_{I}$, relative to that of the metal layer $h_{I}$, on the limit strains (Fig. 9a and b) and on the necking band orientations (Fig. 9c and d) when the bifurcation analysis is used. When the initial thickness $H_{I}$ of the elastomer layer is equal to 0 , the bifurcation results of a freestanding metal layer (Fig. 4) are naturally recovered. For both adopted plasticity theories (flow theory and deformation theory), the effect of the elastomer layer is to shift the FLD monotonically upwards, and thus to enhance the ductility of the bilayer. The larger the relative thickness $H_{I} / h_{I}$, the more significant the retardation of necking occurrence. This result demonstrates the practical interest in the use of elastomer substrates to increase the necking limit of metal components. It must be noted that the plots of Fig. $9 \mathrm{~b}$ are very similar to those reported in Jia and $\mathrm{Li}$ (2013). In Fig. 9c, the necking band orientation $\theta$ is plotted versus the strain path $\rho$ when the mechanical behavior of the metal layer is modeled by the flow theory. In this case, the presence of an elastomer layer, under the condition of perfect adherence, does not affect the necking band orientation, which follows the evolution rule given by Eq. (57). For this reason, the different plots in Fig. 9c are indistinguishable. This result of necking band orientation is also consistent with that of a freestanding metal layer, because for rigid-plastic flow theory, the band orientation is determined by a line of zero extension rate (Hill, 1952), whose orientation is found only to depend on the strain path $\rho$ and not on the material parameters (e.g., hardening). Another remarkable difference with the deformation theory is that the band orientation starts to incline (to deviate from zero), in the case of flow theory, as soon as the strain path $\rho$ becomes negative (see Fig. 9c). On the other hand, when deformation theory is used, the localization band remains perpendicular to the major strain direction when the strain path

ratio $\rho$ is between 0 (plane strain tension) and a negative threshold value $\rho_{c}$. This threshold value depends on the elastomer relative thickness (e.g., $\rho_{c}=-0.07,-0.08,-0.1$ and -0.12 when $H_{I} / h_{I}=0$, 1,2 and 3 , respectively). Then, this band orientation starts to increase when $\rho$ is inferior to $\rho_{c}$. Note also that this band orientation, predicted with the deformation theory, depends on the elastomer relative thickness $H_{I} / h_{I}$, as revealed by Fig. 9d, and also on the strain hardening parameter $N$. This latter dependence on the hardening parameter has already been shown for a freestanding metal layer (see Fig. 4c and d). 


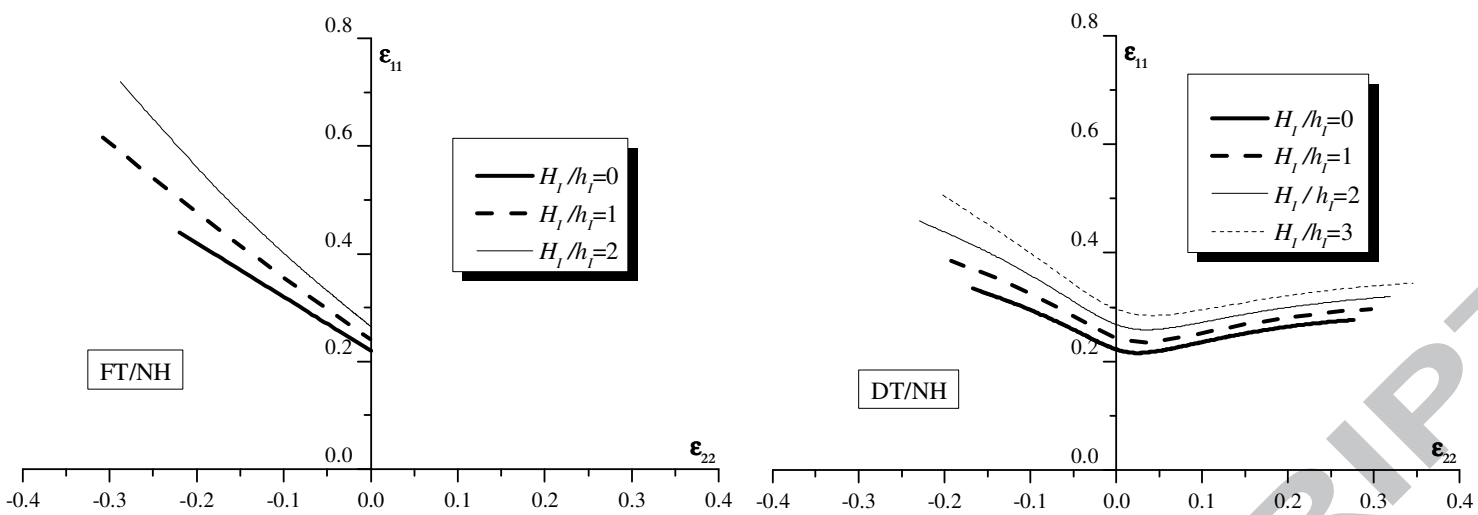

(a)

(b)

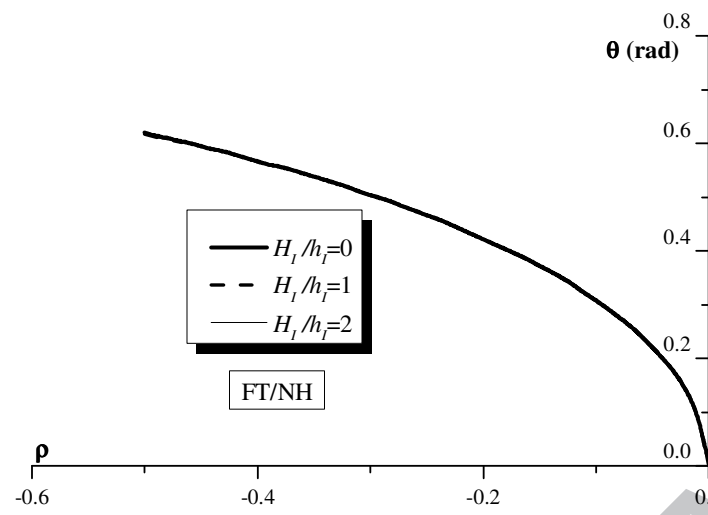

(c)

(d)

Fig. 9. Effect of the elastomer relative thickness on the FLDs and on the necking band orientations, as predicted by the bifurcation theory, for a metal/elastomer bilayer; comparison between FT/NH and DT/NH bilayers ( $N=0.22, \mu=22 \mathrm{MPa}$ ): (a) FLDs for FT/NH bilayer; (b) FLDs for DT/NH bilayer; (c) Necking band orientations for FT/NH bilayer; (d) Necking band orientations for DT/NH bilayer.

In order to further understand the effect of the elastomer relative thickness on the necking retardation, Fig. 10 depicts the evolution of the limit strain $\varepsilon_{11}^{L}$ as a function of the initial thickness ratio $H_{I} / h_{I}$ for the two material combinations and for different strain paths. From this figure, it is clear that the limit strain $\varepsilon_{11}^{L}$ increases almost linearly and slowly with the initial thickness ratio $H_{I} / h_{I}$, for the different strain paths reported, except in the case $\rho=-0.5$. In this latter case and for both material combinations, the limit strain increases rapidly, a trend that is sensibly more pronounced for the flow theory modeling. Furthermore, the two plasticity theories provide the same predictions for the limit strain and its evolution in the case of plane strain tension $(\rho=0)$. This result has also been observed in the case of a freestanding metal layer (see Section 5.1). 


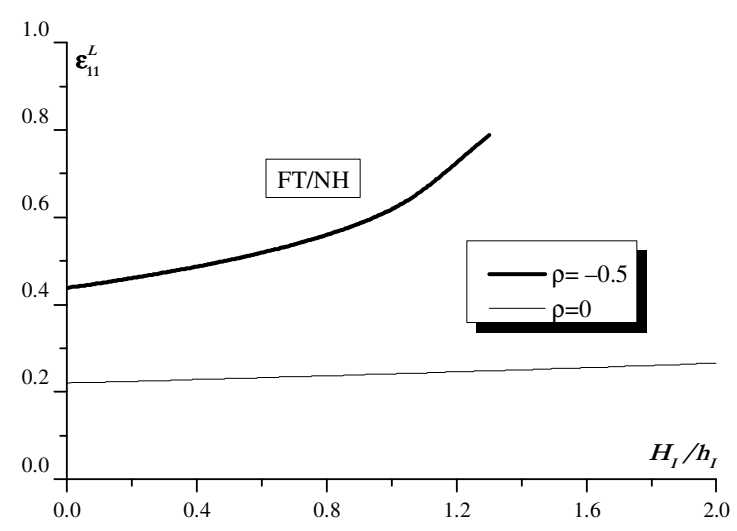

(a)

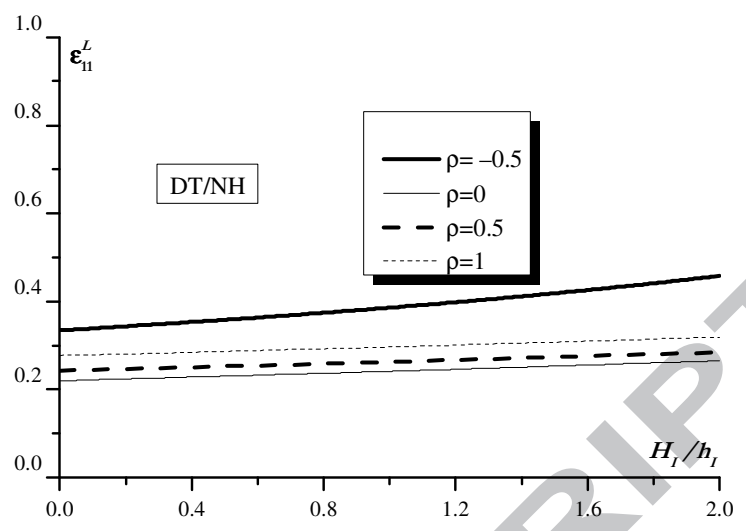

(b)

Fig. 10. Evolution of the limit strain as a function of the initial thickness ratio for different strain paths, as predicted by the bifurcation theory, for a metal/elastomer bilayer; comparison between FT/NH and DT/NH bilayers ( $N=0.22, \mu=22 \mathrm{MPa}$ ): (a) FT/NH bilayer; (b) DT/NH bilayer.

It is well-known from the literature (Xue and Hutchinson, 2007; Jia and Li, 2013) that the neck retardation is tied to the fact that under biaxial loading, the tangent modulus of the elastomer remains unchanged, or increases, while the tangent modulus of the metal layer steadily decreases. When the band orientation is assumed to be equal to 0 (hence the normal $\vec{n}$ to the band is equal to $\{1,0\}$ ), the acoustic tensor $\underline{C}$ (associated with a single material) can be expressed as a function of the components of the corresponding analytical tangent modulus $\mathscr{L}$ (which is itself function of the instantaneous modulus $\underline{L}$ and some stress components, see Eqs. (25)-(27)) by the following expression:

$$
\underline{C}=\left(\begin{array}{cc}
C_{11} & 0 \\
0 & C_{22}
\end{array}\right)=\left(\begin{array}{cc}
\mathscr{L}_{1111} & 0 \\
0 & \mathscr{L}_{1221}
\end{array}\right)=\left(\begin{array}{cc}
L_{11}-\sigma_{11} & 0 \\
0 & L_{s}-\frac{1}{2}\left(\sigma_{22}-\sigma_{11}\right)
\end{array}\right)
$$

With this specific form for the acoustic tensor, the bifurcation occurs as soon as one of the components $C_{11}$ or $C_{22}$ becomes equal to zero for the first time. Under this condition, the major strain would represent the critical strain.

In order to check the dependence of the neck retardation on the evolution of the tangent modulus, the above components $C_{11}$ and $C_{22}$ of the acoustic tensor are plotted in Fig. 11 versus the major strain component $\varepsilon_{11}$ for the different materials, in the case of uniaxial tension $(\rho=-0.5)$ and plane strain tension ( $\rho=0$ ), and when the band orientation $\theta$ is fixed to 0 . To clearly show the difference between the different models, the components $C_{11}$ and $C_{22}$ are normalized by $210 \mathrm{GPa}$ in the case of flow theory and deformation theory (this value corresponds to the Young modulus of usual steels) and by $66 \mathrm{MPa}$ in the case of neo-Hookean model (this value corresponds to the Young modulus of the elastomer used here). In view of these results, some conclusions can be drawn:

- When the neo-Hookean model is used, the component $C_{22}$ increases (Fig. 11b and d). However, the component $C_{11}$ decreases slowly at the beginning of the loading and then increases (Fig. 11a 
and c). Considering the evolution of these components and the expression (23) for the instantaneous modulus of the neo-Hookean model, we can conclude that necking can never occur in the elastomer layer alone.

- The components $C_{11}$ and $C_{22}$ decrease rather quickly when the deformation theory is used for the different strain paths (Fig. 11a, b, c and d). Furthermore, $C_{11}$ becomes equal to 0 when the major strain $\varepsilon_{11}$ is equal to 0.55 (resp. 0.22) for the uniaxial tension path (resp. plane strain tension), (see Fig. 11a and c). In the latter case of plane strain tension, the predicted critical strain $\varepsilon_{11}^{*}=0.22$ corresponds to the localization limit strain that is associated with the onset of necking for an all-metal single layer modeled with the deformation theory.

- The component $C_{11}$ has the same evolution for both the deformation theory and flow theory in the case of plane strain tension (Fig. 11c). This means that the localization limit strains predicted by both plasticity theories are identical, in plane strain tension, a feature that has been already discussed previously.

- The components $C_{11}$ and $C_{22}$, associated with flow theory, remain strictly positive during the uniaxial tensile loading (Fig. 11a and b), at least in the strain range considered $\left(0<\varepsilon_{11}<0.6\right)$. Therefore, bifurcation cannot occur at realistic strain values for this band orientation $(\theta=0)$. Obviously, for other values of the band orientation, it is possible to reach bifurcation at realistic strain values (see Fig. 8c).

- For all of the above-mentioned reasons, we can observe easily that the components $C_{11}$ and $C_{22}$ associated with the metal/elastomer bilayer decrease with the loading, but at a rate lower than that for a freestanding metal layer alone. Accordingly, the choice of bonding an elastomer substrate to a metal layer in order to retard the necking occurrence and thus to enhance its ductility becomes understandable.

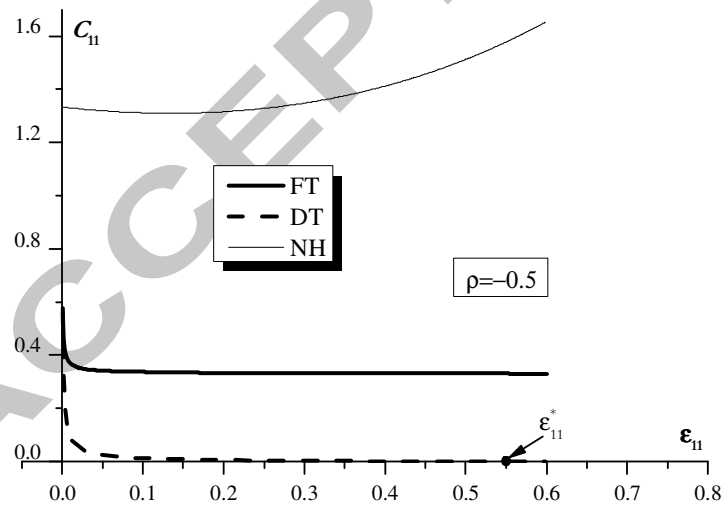

(a)

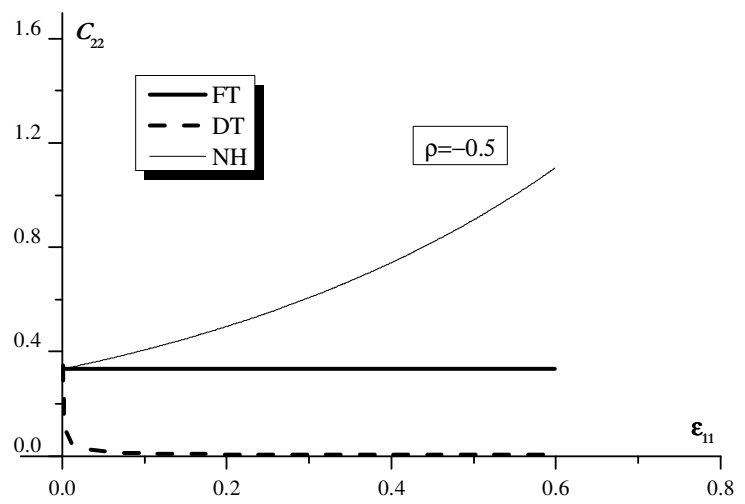

(b) 


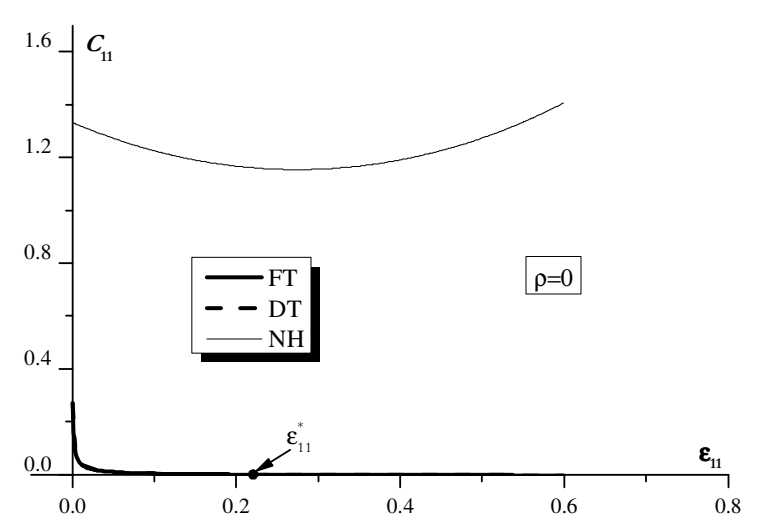

(c)

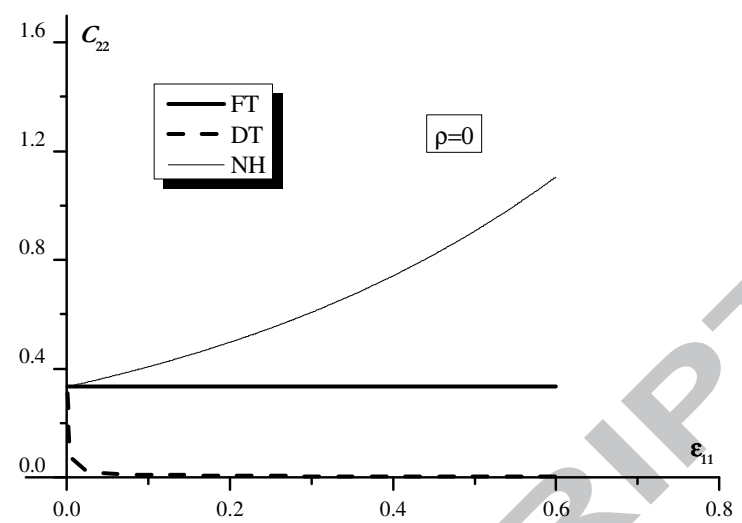

(d)

Fig. 11. Evolution of the normalized components of the acoustic tensor (associated with different materials taken separately) versus the major strain for a band orientation $\theta=0$; comparison between

flow theory, deformation theory and neo-Hookean model ( $N=0.22, \mu=22 \mathrm{MPa}$ ): (a) $C_{11}$ for

$$
\rho=-0.5 \text {; (b) } C_{22} \text { for } \rho=-0.5 \text {; (c) } C_{11} \text { for } \rho=0 ; \text { (d) } C_{22} \text { for } \rho=0 \text {. }
$$

\subsection{2. $M-K$ analysis results}

The effect of an initial geometric imperfection $\xi_{I}$ (in the metal layer) on the necking limit strain of the bilayer is investigated in Fig. 12. Two different values for the initial geometric imperfection are considered, which are given by $\xi_{I}=10^{-2}$ and $2 \times 10^{-2}$. As can be seen, and similarly to the bifurcation analysis, the presence of an elastomer layer allows an enhancement of the necking limit strain for both initial imperfection values. Interestingly, the comparison between Fig. 12a and Fig. 12c or between Fig. 12b and Fig. 12d reveals that the ductility lost due to the presence of an initial imperfection can be caught up by bonding an elastomer layer. The comparison between Fig. 9 and Fig. 12 indicates that the necking limit strains determined from the bifurcation analysis set an upper bound to the FLDs given by the M-K approach.

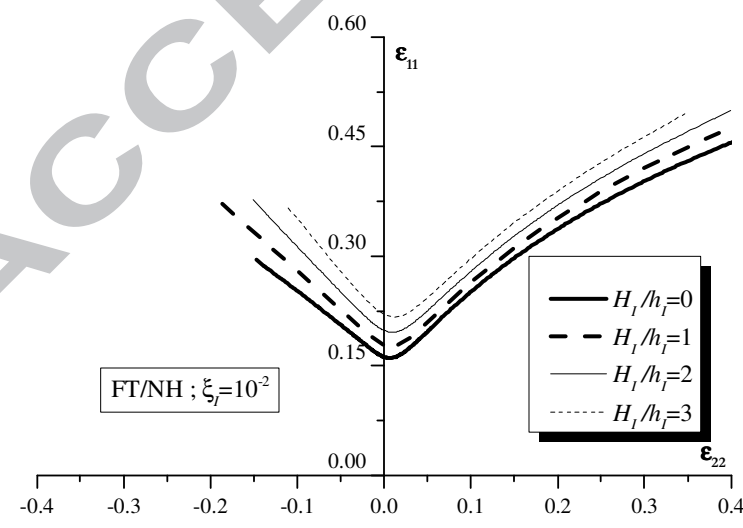

(a)

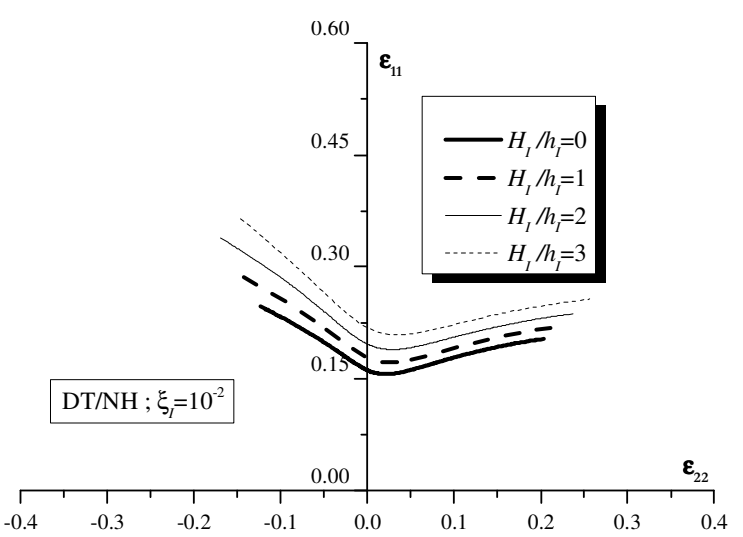

(b) 


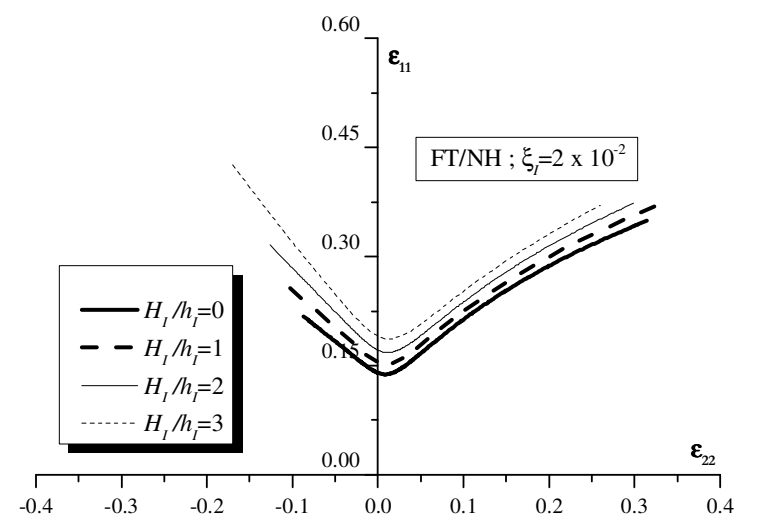

(c)

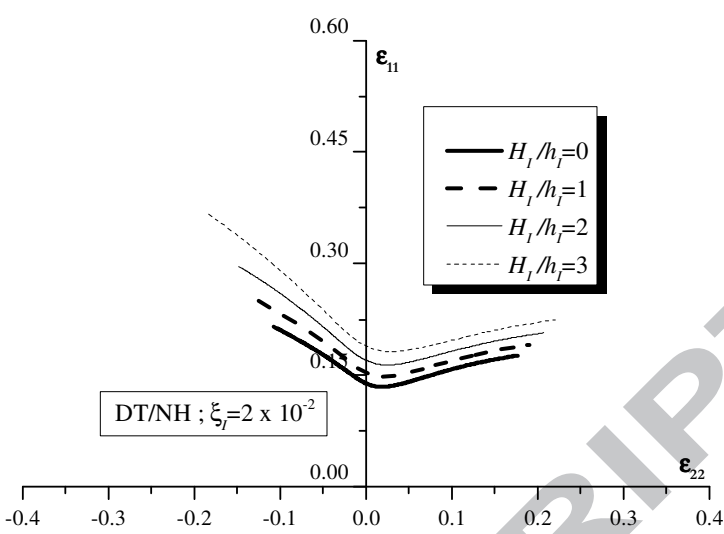

(d)

Fig. 12. Effect of the thickness ratio on the FLDs predicted by the $M-K$ approach, for a metal/elastomer bilayer; comparison between FT/NH and DT/NH bilayers $(\mathrm{N}=0.22, \mu=22 \mathrm{MPa})$ : (a) FT/NH bilayer $\left(\xi_{I}=10^{-2}\right)$; (b) DT/NH bilayer $\left(\xi_{I}=10^{-2}\right)$; (c) FT/NH bilayer $\left(\xi_{I}=2 \times 10^{-2}\right)$; (d)

$$
\text { DT/NH bilayer }\left(\xi_{I}=2 \times 10^{-2}\right) \text {. }
$$

The effect of the initial geometric imperfection $\xi_{I}$ on the shape and the location of the FLDs of the elastomer/metal bilayer is depicted in Fig. 13. The initial thickness ratio $H_{I} / h_{I}$ is taken equal to 1 . The same trends observed in Fig. 6, when an all-metal single layer has been studied, are observed for the bilayer: an increase of the initial geometric imperfection $\xi_{I}$ reduces considerably the critical strains for localization of the bilayer. Also, in a similar way, the $\mathrm{M}-\mathrm{K}$ predictions tend towards the bifurcation results in the limit of a vanishing size for the initial imperfection.

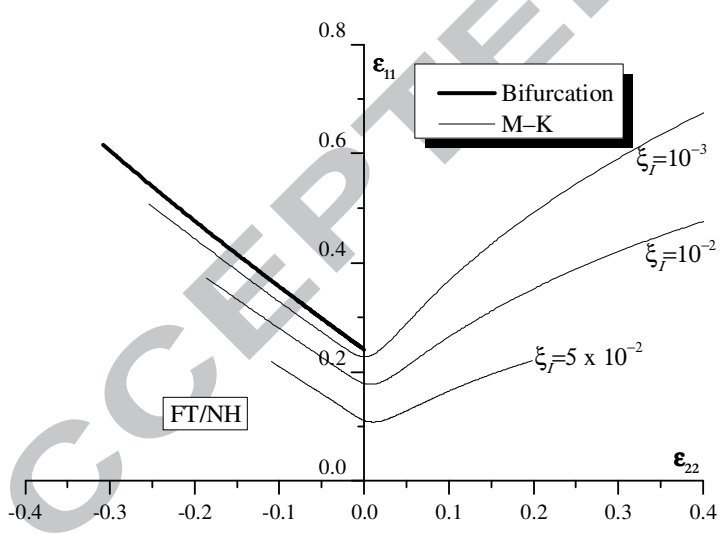

(a)

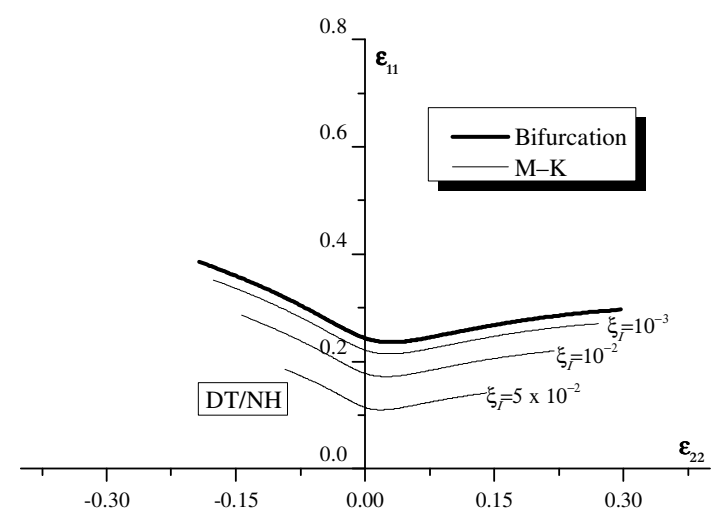

(b)

Fig. 13. Effect of $\xi_{I}$ on the shape and the location of the FLDs for a metal/elastomer bilayer; comparison between FT/NH and DT/NH bilayers $\left(H_{I} / h_{I}=1, N=0.22, \mu=22 \mathrm{MPa}\right)$ : (a) FT/NH bilayer; (b) DT/NH bilayer.

Fig. 14 gives the evolution of the critical strain $\varepsilon_{11}^{*}$ with both the initial and current band orientation of the bilayer in the case of uniaxial tension $(\rho=-0.5)$. The initial thickness ratio $H_{I} / h_{I}$ is taken equal 
to 1 . These results are very similar to those of Fig. 8: the critical strains are sensitive to variations of the band orientation, especially when the combination FT/NH bilayer is considered.

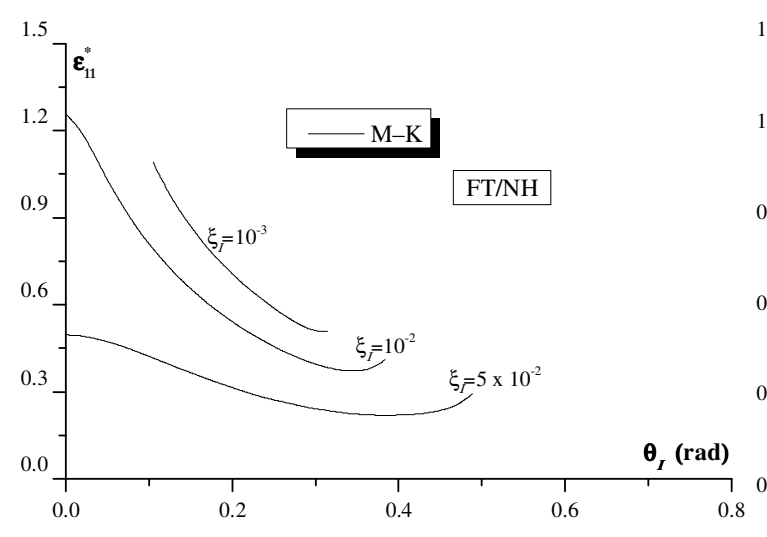

(a)

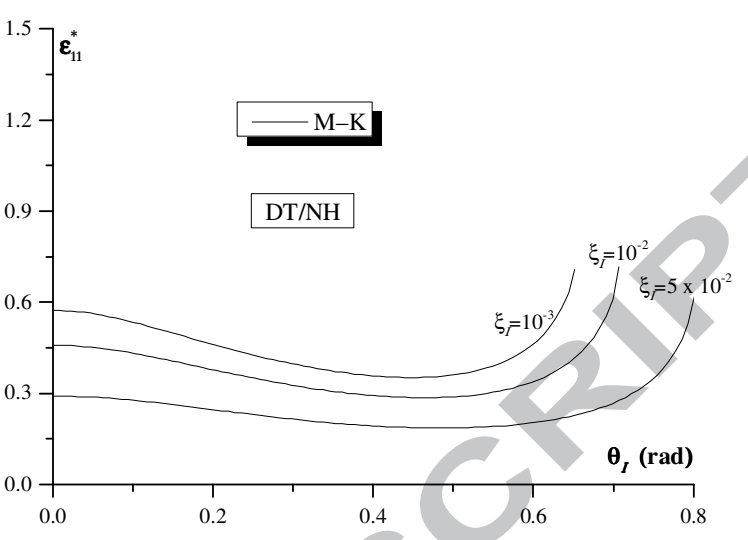

(b)

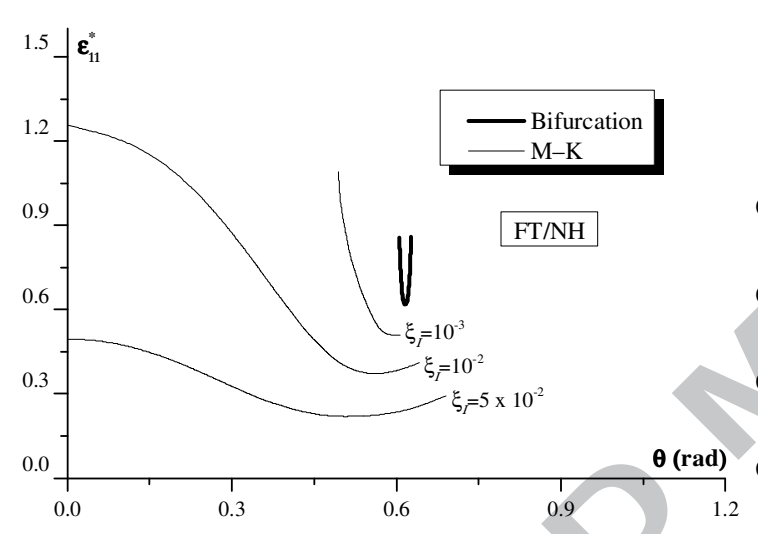

(c)

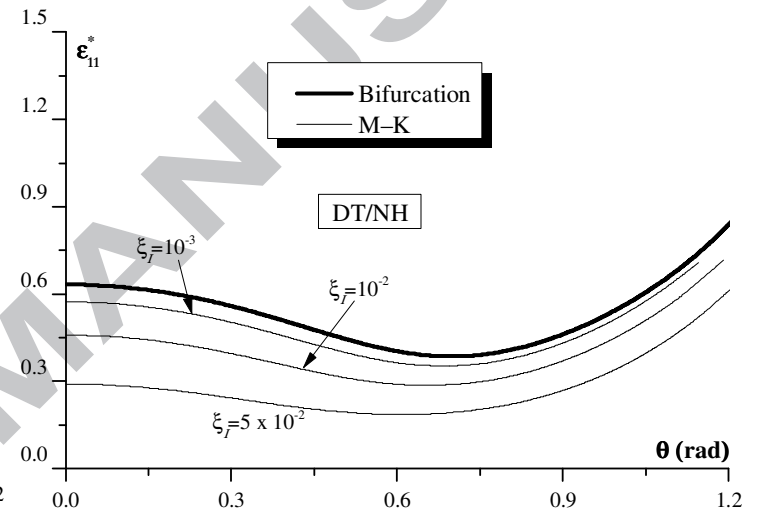

(d)

Fig. 14. Critical strain as a function of necking band orientation for a metal/elastomer bilayer; comparison between FT/NH and DT/NH bilayers $\left(\rho=-0.5, H_{I} / h_{I}=1, N=0.22, \mu=22 \mathrm{MPa}\right)$ : (a) Initial orientation for FT/NH bilayer; (b) Initial orientation for DT/NH bilayer; (c) Current orientation for FT/NH bilayer; (d) Current orientation for DT/NH bilayer.

\section{Conclusions}

In this paper, several numerical schemes and tools have been developed to predict the onset of localized necking in substrate-supported metal layers. The mechanical behavior of the metal is defined by the rigid-plastic flow theory or the deformation theory of plasticity. However, a neo-Hookean law is used to model the elastomer behavior. The metal and the elastomer layers are assumed to be isotropic, incompressible and strain-rate independent. The materials composing the bilayer remain bonded and are such that material damage does not occur prior to necking. Hence, other failure phenomena different from necking (such as damage, interfacial delamination) are not considered in this work. The numerical development is general enough to be used for other behavior models (for the metal or the elastomer) and to be extended to the study of multilayers. The onset of necking is predicted by using both the bifurcation and the M-K analyses. 
From the simulation results, key findings can be summarized below:

- The general trend for freestanding metal layers and bilayers remains that the largest limit strains are predicted when the mechanical behavior of the metal layer is modeled by the flow theory. This conclusion holds whatever the localization approach: bifurcation theory or M-K analysis.

- The presence of an elastomer layer enhances substantially the necking limit of the metal/elastomer bilayer. This observation is common to bilayers with or without geometric imperfection. This neck retardation is due to the mechanical constraint of the substrate to the metal deformation. For the bifurcation analysis, this conclusion is easily understandable considering the form and the evolution of the acoustic tensor components of each layer.

- Similar to the case of an all-metal single layer, the necking band of the bilayer for positive strain paths $(\rho \geq 0)$ is found to be perpendicular to the direction of major strain. This result is true for both plasticity theories and both localization approaches: the bifurcation theory (when applicable) and the M-K analysis.

- For negative strain paths $(\rho \leq 0)$ and when the combination of FT/NH bilayer is considered, the necking band orientation is found to be independent of the elastomer relative thickness and of strain hardening parameters; it only depends on the strain path parameter $\rho$ in the same way as for a freestanding metal layer modeled by the flow theory.

- For both plasticity theories, the limit strains predicted by the bifurcation analysis set an upper bound to those given by the imperfection approach. Indeed, the FLDs determined by the M-K analysis tend towards those predicted by the bifurcation analysis in the limit of a vanishing size for the initial imperfection. This observation is natural considering the similarity of the mathematical formulations of the two approaches ( $\mathrm{M}-\mathrm{K}$ and bifurcation): if the amount of initial imperfection is set equal to 0 in the imperfection model, the problem is reduced to the bifurcation analysis. This conclusion is valid for a freestanding metal layer as well as for an elastomer-supported metal layer.

To summarize, the proposed numerical schemes represent a powerful tool that could be used to help design and select elastomer-supported metal layers that provide optimal mechanical properties (good ductility, reduced mass...). 


\section{Appendix A. Algorithm for the computation of the critical strains}

This algorithm is based on the time integration of the evolution problem composed of Eqs. (16), (17), (36) and (39). This time integration proceeds by discretizing the deformation history in time and numerically integrating these equations over each typical time step $[\tau, t]$. For this purpose, we assume that the following quantities are known at $\tau: \underline{F}_{\tau}^{B}, \underline{F}_{\tau}^{0}, \underline{\sigma}_{0}^{B}(U), \underline{\sigma}_{\tau}^{B}(L), \underline{\sigma}_{\tau}^{0}(U), \underline{\sigma}_{\tau}^{0}(L)$. The aim of this algorithm is to compute these same quantities at $t$ and then to determine $\varepsilon_{11}^{0}$. We will show that the solution of the evolution problem can be reduced to the determination of $\beta_{1}$ and $\beta_{2}$. In order to simplify the notations, subscript $t$ indicating the time dependence will be omitted in the following, with the understanding that all variables are evaluated at $t$, unless otherwise indicated.

For each time increment $[\tau, t]$, the following steps are carried out:

- Step 1: Assuming that $\vec{n}$ is equal to $\vec{n}_{\tau}$, determine $\underline{G}^{0}$ and $\underline{G}^{B}$ as functions of $\beta_{1}$ and $\beta_{2}$, as demonstrated in subsection 4.2.2.1. This assumption permits to avoid solving the non-linear differential equation obtained by the combination of Eqs. (55) and (56), without loss of accuracy for the final result.

- Step 2: Express $\underline{F}^{0}$ and $\underline{F}^{B}$ as functions of $\beta_{1}$ and $\beta_{2}$ :

$$
\underline{F}^{0}=\exp \left((t-\tau) \underline{G}^{0}\right) \underline{F}_{\tau}^{0} ; \underline{F}^{B}=\exp \left((t-\tau) \underline{G}^{B}\right) \underline{F}_{\tau}^{B}
$$

- Step 3: By using $K$ and $N$, and $\underline{G}^{0}$ and $\underline{G}^{B}$, use Eqs. (16) and (17) $)_{1}$ to express the final values of $\underline{\sigma}^{B}(U)$ and $\underline{\sigma}^{0}(U)$ in the metal layer as functions of $\beta_{1}$ and $\beta_{2}$.

- Step 4: By using $\mu$ and $\underline{F}^{0}$ and $\underline{F}^{B}$, as determined above, use Eqs. (16) and (17) $)_{3}$ to express the final values of $\underline{\sigma}^{B}(L)$ and $\underline{\sigma}^{0}(L)$ in the elastomer layer as functions of $\beta_{1}$ and $\beta_{2}$.

- Step 5: Update the layer thicknesses inside and outside the band by the following relations:

$$
\begin{aligned}
& h^{0}=h_{\tau}^{0} \exp \left(-(1+\rho)(t-\tau) \dot{\varepsilon}_{11}^{0}\right) \quad ; \quad H^{0}=H_{\tau}^{0} \exp \left(-(1+\rho)(t-\tau) \dot{\varepsilon}_{11}^{0}\right) \\
& h^{B}=h_{\tau}^{B} \exp (-(t-\tau)) \quad ; \quad H^{B}=H_{\tau}^{B} \exp (-(t-\tau))
\end{aligned}
$$

where the only unknown in Eq. (A.2) is $\dot{\varepsilon}_{11}^{0}$, which is function of $\beta_{1}$ and $\beta_{2}$.

- Step 6: Analyzing the different quantities computed in Steps 1 to 5, it is clear that the equilibrium equation (39) becomes function only of $\beta_{1}$ and $\beta_{2}$. This equation is then solved to determine the values of $\beta_{1}$ and $\beta_{2}$ and then to determine $\varepsilon_{11}^{0}$.

Localized necking in the groove is said to occur when the necking criterion:

$$
\left|\dot{\varepsilon}_{33}^{B}\right|>10\left|\dot{\varepsilon}_{33}^{0}\right|
$$

is satisfied. Therefore, the implicit incremental algorithm (defined by Steps 1 to 6) is repeated up to the point at which the necking criterion (A.3) is satisfied. The factor 10 in (A.3) is rather arbitrary and any other relatively large positive number can be used. It is demonstrated that the effect of this factor 
on the predicted necking strains is minimal, since the ratio $\left|\dot{\varepsilon}_{33}^{B}\right| /\left|\dot{\varepsilon}_{33}^{0}\right|$ increases very steeply towards infinity at incipient necking.

\section{References}

Abed-Meraim, F., 2009. Contributions à la prédiction d'instabilités de type structure et matériau : modélisation de critères et formulation d'éléments finis adaptés à la simulation des structures minces. HDR, Université Paul Verlaine de Metz, France.

Alaca, B.E., Saif, M.T.A., Sehitoglu, H., 2002. On the interface debond at the edge of a thin film on a thick substrate. Acta Materialia 50, 1197-1209.

Amirkhizi, A.V., Isaacs, J., McGee, J., Nemat-Nasser, S., 2006. An experimentally-based viscoelastic constitutive model for polyurea including pressure and temperature effects. Philosophical Magazine 86, 58475866.

Ayoub, G., Zaïri, F., Naït-Abdelaziz, M., Gloaguen, J.M., Kridli, G., 2014. A visco-hyperelastic damage model for cyclic stress-softening, hysteresis and permanent set in rubber using the network alteration theory. International Journal of Plasticity 54, 19-33.

Baghani, M., Naghdabadi, R., Arghavani, J., Sohrabpour, S., 2012. A thermodynamically-consistent 3 D constitutive model for shape memory polymers. International Journal of Plasticity 35, 13-30.

Barlat, F., 1989. Forming limit diagrams-predictions based on some microstructural aspects of materials. In: Wagoner, R.H., Chan, K.S., Keeler, S.P. (Eds.), Forming Limit Diagrams: Concepts, Methods and Applications. The Minerals, Metals and Materials Society, Warrendale, PA, 275-302.

Baudelet, B., 1984. Prediction of forming limit diagrams for deep drawing. Agard Lect. Ser. 137 (3), 1-22.

Bouvard, J.L., Francis, D.K., Tschopp, M.A., Marin, E.B., Bammann, D.J., Horstemeyer, M.F., 2013. An internal state variable material model for predicting the time, thermomechanical, and stress state dependence of amorphous glassy polymers under large deformation. International Journal of Plasticity 42, 168-193.

Chiu, S.L., Leu, J., Ho, P.S., 1994. Fracture of metal-polymer line structures. I. Semiflexible polyimide. Journal of Applied Physics 76, 5136-5142.

Cordill, M.J., Taylor, A., Schalko, J., Dehm, G., 2010. Fracture and delamination of chromium thin films on polymer substrates. Metall. Mater. Trans. A 41A, 870-875.

Cotton, D.P.J., Graz, I.M., Lacour, S.P., 2009. A multifunctional capacitive sensor for stretchable electronic skins. IEEE Sens. J. 9, 2008-2009.

Eyckens, P., Van Bael, A., Van Houtte, P., 2009. Marciniak-Kuczynski type modelling of the effect of ThroughThickness Shear on the forming limits of sheet metal. International Journal of Plasticity 25, 2249-2268.

Franz, G., Abed-Meraim, F., Lorrain, J.-P., Ben Zineb, T., Lemoine, X., Berveiller, M., 2009. Ellipticity loss analysis for tangent moduli deduced from a large strain elastic-plastic self-consistent model. International Journal of Plasticity 25, 205-238. 
Franz, G., Abed-Meraim, F., Berveiller, M., 2013. Strain localization analysis for single crystals and polycrystals: Towards microstructure-ductility linkage. International Journal of Plasticity 48, 1-33.

Goodwin, G.M., 1968. Application of strain analysis to sheet metal forming problems in press shop. Metallurgia Italiana 60 (8), 767-774.

Graudejus, O., Jia, Z., Li, T., Wagner, S., 2012. Size-dependent rupture strain of elastically stretchable metal conductors. Scr. Mater. 66, 919-922.

Haddag, B., Abed-Meraim, F., Balan, T., 2009. Strain localization analysis using a large deformation elasticplastic model coupled with damage. Int. J. Plast. 25, 1970-1996.

Hill, R., 1950. Mathematical Theory of Plasticity. Oxford University Press, Oxford.

Hill, R., 1952. On discontinuous plastic states, with special reference to localized necking in thin sheets. J. Mech. Phys. Solids 1, 19-30.

Hill, R., 1970. Constitutive inequalities for isotropic elastic solids under finite strain. Proc. R. Soc. London Ser. A $314,457-472$.

Hollomon, J.H., 1945. Tensile deformation. Transactions of AIME 162, 268-290.

Hommel, M., Kraft, O., 2001. Deformation behavior of thin copper films on deformable substrates. Acta Materialia 49, 3935-3947.

Hunter, S.C., 1979. Some exact solutions in the theory of finite elasticity for incompressible neo-hookean materials. Int. J. Mech. Sci. 21, 203-211.

Hutchinson, J.W., Neale, K.W., Needleman, A., 1978a. Sheet necking-I. Validity of plane stress assumptions of the long-wavelength approximation. In: Koistinen, D.P., Wang, N.M. (Eds.), Mechanics of Sheet Metal Forming. Plenum, 111-126.

Hutchinson, J.W., Neale, K.W., 1978b. Sheet necking-II. Time-independent behavior. In: Koistinen, D.P., Wang, N.M. (Eds.), Mechanics of Sheet Metal Forming. Plenum, 127-153.

Hutchinson, J.W., Neale, K.W., 1981. Finite Strain $J_{2}$ Deformation Theory. In Proceedings of the IUTAM Symposium on Finite Elasticity edited by D. E. Carlson and R. T. Shield, Martinus Nijhoff Publishers, Netherlands, 238-247.

Jia, Z., Li, T., 2013. Necking limit of substrate-supported metal layers under biaxial in-plane loading. International Journal of Plasticity 51, 65-79.

Keeler, S.P., Backofen, W.A., 1963. Plastic instability and fracture in sheets stretched over rigid punches. Trans. ASM 56, 25-48.

Khan, A.S., Baig, M., 2011. Anisotropic responses, constitutive modeling and the effects of strain-rate and temperature on the formability of an aluminum alloy. International Journal of Plasticity 27, 522-538.

Lacour, S.P., Jones, J., Wagner, S., Li, T., Suo, Z., 2005. Stretchable interconnects for elastic electronic surfaces. Proc. IEEE 93, 1459-1467.

Li, J., Carsley, J.E., Stoughton, T.B., Hector Jr., L.G., Hu, S.J., 2013. Forming limit analysis for two-stage forming of 5182-O aluminum sheet with intermediate annealing. International Journal of Plasticity 45, 21-43. 
Marciniak, Z., Kuczynski, K., 1967. Limit strains in the processes of stretch-forming sheet metal. International Journal of Mechanical Sciences 9, 609-620.

Narayanasamy, R., Sathiya Narayanan, C., 2005. Forming limit diagram for interstitial free steels Part I. Materials Science and Engineering A 399, 292-307.

Rajagopal, K.R., 1998. On a class of elastodynamic motions in a neo-hookean elastic solid. Int. J. Non-Linear Mechanics 33, 397-405.

Rice, J.R., 1976. The localization of plastic deformation. In: 14th International Congress of Theoretical and Applied Mechanics, 207-220.

Rudnicki, J.W., Rice, J.R., 1975. Conditions for localization of deformation in pressure-sensitive dilatant materials. J. Mech. Phys. Solids 23, 371-394.

Saje, M., Pan, J., Needleman, A., 1982. Void nucleation effects on shear localization in porous plastic solids. Int. J. Fract. 19, 163-182.

Smith, P.E., Lee, D., 1998. Determination of forming limits for aluminum alloys. SAE \#982375.

Stören, S., Rice, J.R., 1975. Localized necking in thin sheets. Journal of the Mechanics and Physics of Solids 23, $421-441$.

Strano, M., Colosimo, B.M., 2006. Logistic regression analysis for experimental determination of forming limit diagrams. International Journal of Machine Tools \& Manufacture 46, 673-682.

Uchida, M., Tada, N., 2013. Micro-, meso- to macroscopic modeling of deformation behavior of semi-crystalline polymer. International Journal of Plasticity 49, 164-184.

Xue, Z.Y., Hutchinson, J.W., 2007. Neck retardation and enhanced energy absorption in metal/elastomer bilayers. Mechanics of Materials 39, 473-487.

Xue, Z.Y., Hutchinson, J.W., 2008. Neck development in metal/elastomer bilayers under dynamic stretchings. International Journal of Solids and Structures 45, 3769-3778.

Zhang, L., Wang, J., 2012. Modeling the localized necking in anisotropic sheet metals. International Journal of Plasticity 39, 103-118. 


\section{Research highlights}

- Efficient numerical tools are developed to predict localized necking in elastomer/metal bilayers.

- The predictions are based on two necking limit criteria and two constitutive frameworks.

- The ductility of freestanding metal layers is compared to that of substrate-supported metal layers.

- The impact of the behavior of the metal layer on the ductility of the bilayer is investigated.

- The combined effect of initial imperfection and elastomer relative thickness is analyzed. 